\title{
LARGE SCALE SINGULARLY PERTURBED BOUNDARY VALUE PROBLEMS
}

\author{
M. Kathirkamanayagan and G.S. Ladde \\ Department of Mathematics \\ The University of Texas at Arlington \\ Arlington, Texas 76019
}

\begin{abstract}
In this paper an alternative approach to the method of asymptotic expansions for the study of a singularly perturbed linear system with multiparameters and multiple time scales is developed. The method consists of developing a linear non-singular transformation that transforms an arbitrary $\mathrm{n}$-time scale system into a diagonal form. Furthermore, a dichotomy transformation is employed to decompose the faster subsystems into stable and unstable modes. Fast, slow, stable and unstable modes decomposition processes provide a modern technique to find an approximate solution of the original system in terms of the solution of an auxiliary system. This method yields a constructive and computationally attractive way to investigate the system.
\end{abstract}

Key Words: Diagonalization process, decoupled system, dichotomy transformation, fast sub-system, fundamental matrix solution, multiparameter, optimal control, stable and unstable systems.

AMS Subject Classification: $34 \mathrm{~B} 05$ and 34E15 


\section{Introduction}

Singular perturbations of two point boundary value problem is an active subject of research with long history. By employing the asymptotic expansion of such systems under strong conditions on the coefficient matrices, existence, uniqueness and approximations of solutions of such systems are studied in $[12,13,14]$. In $[2,5,15,16]$, under less demanding conditions on the coefficient matrices, boundary value problems for two-time scale linear systems are analyzed. Furthermore, the fast and slow mode decomposition approach $[2,9,16]$ provides a modern alternative technique to study the singularly perturbed systems. Moreover using a dichotomy transformation to decompose the faster subsytems into stable and unstble modes yields a constructive and computationally atractive procedure to investigate such problems $[10,15,17,18]$.

In this paper, by following a hierarchial order reduction scheme of a joint multiparameters and multi-time scale singular perturbation of linear system $[6,8,11]$, a linear non-singular transformation which totally decouples an arbitrary n-time scale and multiparameter linear singularly perturbed system, is developed. Furthermore, a dichotomy transformation is employed to decompose the faster subsytems into stable and unstble modes. Sufficient conditions are given for existence and uniqueness of solution of an auxiliary boundary value problem which guarantees the existence, uniqueness and approximation of solution of given boundary value problem. The obtained conditions are given in terms of known coefficient matrices.

This paper is organized as follows: In section 2 , by following the argument $[6,8,11]$, a joint multitime scale multiparameter singularly perturbed two point boundary value problem is formulated. For the sake of convenience and simplicity a few notations are defined in section 3 . These notations will be used throughout this paper. In section 4, by following the decoupling procedure in [6], a totally decoupling process is briefly discussed. Moreover, the asymptotic behaviour and the representation of the transformation in terms of the given coefficient matrices is given. In section 5 , by developing a dichotomy transformation which decomposes the faster subsystems of totally decoupled system into stable and unstable subsystems, the existence, uniqueness and approximation of the original problems are investigated. An example illustrating the decomposing procedures and finding an approximate solution is presented in section 6 . This example exhibits the generalization and extension of the work of Wilde and Kokotovic $[17,18]$.

\section{Problem Formulation}

Consider a linear time-varying system of differential equations

with the boundary condition

$$
\epsilon_{\mathrm{i}}^{\mathrm{j}} \dot{\mathrm{X}}_{\mathrm{ij}}=\sum_{\mathrm{p}=1}^{\mathrm{n}} \sum_{\mathrm{k}=1}^{\mathrm{r} p} A_{\mathrm{ik}}^{\mathrm{j} p}(\mathrm{t}) \mathrm{X}_{\mathrm{pk}}
$$




$$
\sum_{\mathrm{p}=1}^{\mathrm{n}} \sum_{\mathrm{k}=1}^{\mathrm{r} p} \mathrm{Q}_{\mathrm{ik}}^{\mathrm{jp}}(\epsilon) \mathrm{X}_{\mathrm{pk}}(0)+\sum_{\mathrm{p}=1}^{\mathrm{n}} \sum_{\mathrm{k}=1}^{\mathrm{r} p} \mathrm{R}_{\mathrm{ik}}^{\mathrm{jp}}(\epsilon) \mathrm{X}_{\mathrm{pk}}(1)=\mathrm{B}_{\mathrm{i}}^{\mathrm{j}}(\epsilon)
$$

where $\epsilon=\left(\epsilon_{1}^{1} \epsilon_{1}^{2} \ldots \epsilon_{1}^{\mathrm{r}_{1}} \ldots \epsilon_{\mathrm{n}}^{1} \epsilon_{\mathrm{n}}^{2} \ldots \epsilon_{\mathrm{n}}^{\mathrm{r}_{\mathrm{n}}}\right), \mathrm{X}_{\mathrm{ij}} \in \mathrm{R}^{\mathrm{n}_{\mathrm{ij}}}, \mathrm{j} \in \mathrm{J}\left(1, \mathrm{r}_{\mathrm{i}}\right), \mathrm{i} \in \mathrm{J}(1, \mathrm{n}) ; \mathrm{r}_{\mathrm{i}}$ 's are positive integers and $J(a, b)=\{a, a+1, \ldots, b\} ; a, b \in Z^{+} \cup\{0\}, n_{i}=\sum_{j=1}^{r_{i}} n_{i j} ;$ the dimension of the entire system (2.1) is $\mathrm{N}=\sum_{\mathrm{i}=1}^{\mathrm{n}} \mathrm{n}_{\mathrm{i}}$. In (2.1) all the coefficient matrices are continuous on $\mathbb{R}^{+}$and have appropriate dimensions. For $\mathrm{j} \in \mathrm{J}\left(1, \mathrm{r}_{\mathrm{i}}\right)$ and $\mathrm{i} \in \mathrm{J}(1, \mathrm{n})$, parameters $\epsilon_{\mathrm{i}}^{\mathrm{j}}$ 's are positive real numbers. For fixed $i \in J(1, n), \epsilon_{i}^{j}$ 's have the same order with respect to $j$. This means that for fixed $i \in J(1, n)$, the following inequality is valid:

$$
\epsilon_{\mathrm{i}} \leq \frac{\epsilon_{\mathrm{i}}^{\mathrm{j}}}{\epsilon_{\mathrm{i}}^{\mathrm{k}}} \leq \bar{\epsilon}_{\mathrm{i}}, \mathrm{j}, \mathrm{k} \in \mathrm{J}\left(1, \mathrm{r}_{\mathrm{i}}\right)
$$

where $\underline{\epsilon}_{\mathrm{i}}, \bar{\epsilon}_{\mathrm{i}}$ 's are some positive numbers. Furthermore, for any $\mathrm{j} \in \mathrm{J}\left(1, \mathrm{r}_{\mathrm{i}}\right), \epsilon_{\mathrm{i}}^{\mathrm{j}}$ 's have different order with respect to $\mathrm{i}$.

Assumption A 1.2.1:

$$
\left\{\begin{array}{l}
\epsilon_{1}=1 \\
\lim _{\epsilon_{\mathrm{i}} \rightarrow 0} \frac{\epsilon_{\mathrm{i}+1}}{\epsilon_{\mathrm{i}}}=0 \quad \text { for } \mathrm{i} \in \mathrm{J}(2, \mathrm{n}-1)
\end{array}\right.
$$

where $\epsilon_{\mathrm{i}}=\left(\epsilon_{\mathrm{i}}^{1} \epsilon_{\mathrm{i}}^{2} \ldots \epsilon_{\mathrm{i}}{ }^{\mathrm{r}_{\mathrm{i}}}{ }^{\frac{1}{\mathrm{r}_{\mathrm{i}}}}\right.$.

Using (2.4), we can rewrite the system $(2.1)-(2.2)$ as

$$
\epsilon_{\mathrm{i}} \dot{\mathrm{X}}_{\mathrm{i}}=\sum_{\mathrm{j}=1}^{\mathrm{n}} \mathrm{D}_{\mathrm{i}} \mathrm{A}_{\mathrm{ij}}(\mathrm{t}) \mathrm{X}_{\mathrm{j}}
$$

with the boundary condition

$$
\sum_{j=1}^{n} Q_{i j}(\epsilon) X_{j}(0)+\sum_{p=1}^{n} R_{i j}(\epsilon) X_{j}(1)=B_{i}(\epsilon), \quad i \in J(1, n)
$$


where $X_{i}=\left(X_{i 1}^{T} x_{i 2}^{T} \ldots X_{i r_{i}}^{T}\right)^{T} ; i \in J(1, n) ; A_{i p}=\left(A_{i k}^{j p}(t)\right)_{n_{i} x n_{p}} ; Q_{i p}(\epsilon)=\left(Q_{i k}^{j p}(\epsilon)\right)_{n_{i} x_{p}}$,

$\mathrm{R}_{\mathrm{ip}}(\epsilon)=\left(\mathrm{R}_{\mathrm{ik}}^{\mathrm{jp}}(\epsilon)\right)_{\mathrm{n}_{\mathrm{i}} \mathrm{xn} \mathrm{p}}, \mathrm{B}_{\mathrm{i}}(\epsilon)=\left(\mathrm{B}_{\mathrm{i}}^{\mathrm{j}}(\epsilon)\right)_{\mathrm{n}_{\mathrm{ix} 1}} ; \mathrm{i}, \mathrm{p} \in \mathrm{J}(1, \mathrm{n})$.

For simplicity we omit the arqument of the coefficient matrix functions. The matrices in (2.5) are block matrices that are formed in an obvious way from the coefficient matrices in (2.1) with $D_{i}$ 's being defined by:

$$
\mathrm{D}_{\mathrm{i}}=\operatorname{diag}\left\{\left[\frac{\epsilon_{\mathrm{i}}}{\epsilon_{\mathrm{i}}^{1}}\right] \mathrm{I}_{\mathrm{i} 1}\left[\begin{array}{c}
\epsilon_{\mathrm{i}} \\
\frac{\epsilon_{\mathrm{i}}^{2}}{{ }^{2}}
\end{array}\right] \mathrm{I}_{\mathrm{i} 2} \cdots\left[\begin{array}{c}
\epsilon_{\mathrm{i}} \\
\epsilon_{\mathrm{i}} \\
\epsilon_{\mathrm{i}}
\end{array}\right] \mathrm{I}_{\mathrm{ir}}\right\}, \mathrm{i} \in \mathrm{J}(1, \mathrm{n}) .
$$

where $\mathrm{I}_{\mathrm{ir}_{\mathrm{i}}}$ are the identity matrices of appropriate dimensions. In view of (2.3) and (2.4), the elements of the $\mathrm{D}_{\mathrm{i}}$ matrices are bounded, that is,

$$
\stackrel{\epsilon}{\mathrm{i}}_{\mathrm{i}} \leq \frac{\epsilon_{\mathrm{i}}}{\epsilon_{\mathrm{i}}} \leq \widetilde{\epsilon}_{\mathrm{i}}, \mathrm{j} \in \mathrm{J}\left(1, \mathrm{r}_{\mathrm{i}}\right), \mathrm{i} \in \mathrm{J}(1, \mathrm{n})
$$

where the bounds in (2.8) depend on the corresponding bounds in (2.3). Thus system (2.5) $-(2.6)$ is equivalent to multiparameter multiple time scale system $(2.1)-(2.2)$.

\section{Notations and Definitions.}

For the sake of simplicity and convenience to our future presentation, let us introduce the following notations.

For $\mathrm{m} \in \mathrm{J}(0, \mathrm{n}-1), \quad \mathrm{r} \in \mathrm{J}(0, \mathrm{~m}), \quad \mathrm{l} \in \mathrm{J}(\mathrm{r}, \mathrm{n})$

$$
\begin{aligned}
& x_{l r}^{m}=\left(x_{l}^{T}(m), x_{2}^{T}(m), \ldots, x_{l-r}^{T}(m), x_{l-r+l}^{T}(m-1), x_{l-r+2}^{T}(m-2) \ldots, x_{l}^{T}(0)\right), \\
& x_{l r}^{m 0}=x_{l r}^{m}(0), x_{l r}^{m 1}=x_{l r}^{m}(1)
\end{aligned}
$$

where $\quad\left(\mathrm{x}_{1}^{\mathrm{T}}(0) \mathrm{x}_{2}^{\mathrm{T}}(0) \ldots \mathrm{X}_{\mathrm{n}}^{\mathrm{T}}(0)\right)^{\mathrm{T}}=\left(\mathrm{x}_{1}^{\mathrm{T}} \mathrm{x}_{2}^{\mathrm{T}} \ldots \mathrm{x}_{\mathrm{n}}^{\mathrm{T}}\right)^{\mathrm{T}}$

$$
\begin{gathered}
\mathrm{Q}(0, \epsilon)=\left(\mathrm{Q}_{\mathrm{i}, \mathrm{j}}(\epsilon)\right)_{\mathrm{nxn}}, \mathrm{R}(0, \epsilon)=\left(\mathrm{R}_{\mathrm{ij}}(\epsilon)\right)_{\mathrm{nxn}}, \mathrm{B}(\epsilon)=\left(\mathrm{B}_{\mathrm{i}}(\epsilon)\right)_{\mathrm{nx} 1} . \\
\mathrm{I}_{\mathrm{r}}=\mathrm{I}_{\mathrm{n}_{\mathrm{r}} \times \mathrm{n}_{\mathrm{r}}}, \mathrm{I}^{\mathrm{r}}=\mathrm{I}_{\mathrm{i}=1}^{\mathrm{r}} \mathrm{n}_{\mathrm{i}} \times \sum_{\mathrm{i}=1}^{\mathrm{r}} \mathrm{n}_{\mathrm{i}}, \mathrm{I}(\mathrm{r})=\mathrm{I} \sum_{\mathrm{i}=\mathrm{r}}^{\mathrm{n}} \mathrm{n}_{\mathrm{i}} \times \sum_{\mathrm{i}=\mathrm{r}}^{\mathrm{n}} \mathrm{n}_{\mathrm{i}},
\end{gathered}
$$


and for $m \in J(0, n-2)$

$$
\begin{aligned}
& \mathrm{L}_{\mathrm{m}}=\left(\mathrm{L}_{\mathrm{n}-\mathrm{m} 1} \mathrm{~L}_{\mathrm{n}-\mathrm{m} 2} \ldots \mathrm{L}_{\mathrm{n}-\mathrm{m} n-\mathrm{m}-1}\right), \\
& \mathrm{M}_{\mathrm{m}}=\left(\mathrm{M}_{1 \mathrm{n}-\mathrm{m}}^{\mathrm{T}} \mathrm{M}_{2 \mathrm{n}-\mathrm{m}}^{\mathrm{T}} \cdots \mathrm{M}_{\mathrm{n}-\mathrm{m}-1 \mathrm{n}-\mathrm{m}}^{\mathrm{T}}\right)^{\mathrm{T}} .
\end{aligned}
$$

Define

$$
\tilde{A}_{i j}^{m}= \begin{cases}D_{i} A_{i j} & , \text { for } m=0 \text { and } i, j \in J(1, n) \\ \tilde{A}_{i j}^{m-1}+\tilde{A}_{i n-m+1}^{m-1} L_{n-m+1 j}, & \text { for } m \in J(1, n-1) \text { and } i, j \in J(1, n-m) ;\end{cases}
$$

and $\hat{\mathrm{A}}^{\mathrm{m}}$ is defined by

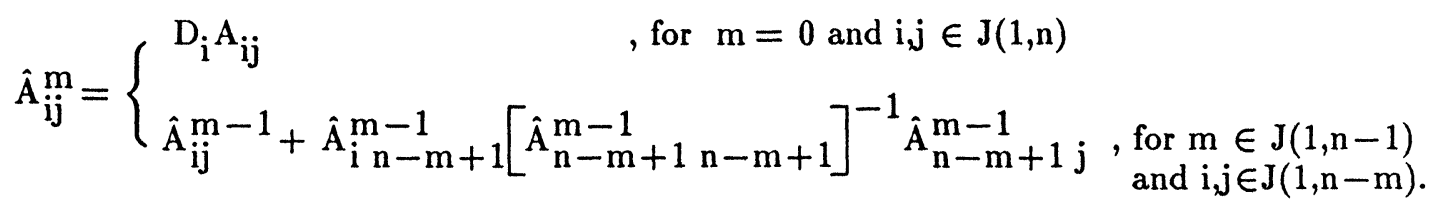

$\hat{A}^{\mathrm{m}}$ can be represented by

$$
\hat{\mathrm{A}}^{\mathrm{m}}=\left[\begin{array}{cc}
\hat{\mathrm{A}}_{11}(\mathrm{~m}) & \hat{\mathrm{A}}_{12}(\mathrm{~m}) \\
\hat{\mathrm{A}}_{21}(\mathrm{~m}) & \hat{\mathrm{A}}_{22}(\mathrm{~m})
\end{array}\right]
$$

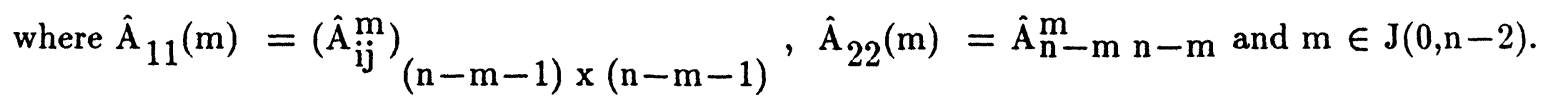

For $\mathrm{m} \in \mathrm{J}(0, \mathrm{n}-2)$, we denote

$$
\mathrm{A}^{\mathrm{m}}=\left[\begin{array}{cc}
\mathrm{A}_{11}(\mathrm{~m}) & \mathrm{A}_{12}(\mathrm{~m}) \\
\frac{1}{\epsilon_{\mathrm{n}-\mathrm{m}} \mathrm{A}_{12}(\mathrm{~m})} & \frac{1}{\epsilon_{\mathrm{n}-\mathrm{m}}} \mathrm{A}_{22}(\mathrm{~m})
\end{array}\right]
$$

where $A_{11}(m)=\left(\frac{1}{\epsilon_{i}} \tilde{A}_{i j}^{m}\right)_{(n-m-1) \times(n-m-1)}$,

$$
\begin{aligned}
& \mathrm{A}_{12}(\mathrm{~m})=\left(\frac{1}{\epsilon_{1}} \tilde{\mathrm{A}}_{1 \mathrm{n}-\mathrm{m}}^{\mathrm{T}} \frac{1}{\epsilon_{2}} \tilde{\mathrm{A}}_{2 \mathrm{n}-\mathrm{m}}^{\mathrm{T}} \cdots \frac{1}{\epsilon_{\mathrm{n}-\mathrm{m}-1}} \tilde{\mathrm{A}}_{\mathrm{n}-\mathrm{m}-1 \mathrm{n}-\mathrm{m}}^{\mathrm{T}}\right)^{\mathrm{T}}, \\
& \mathrm{A}_{21}(\mathrm{~m})=\left(\tilde{\mathrm{A}}_{\mathrm{n}-\mathrm{m} 1}^{\mathrm{m}} \tilde{\mathrm{A}}_{\mathrm{n}-\mathrm{m} 2}^{\mathrm{m}} \cdots \tilde{\mathrm{A}}_{\mathrm{n}-\mathrm{m} \mathrm{n}-\mathrm{m}-1}^{\mathrm{m}}\right), \\
& \mathrm{A}_{22}(\mathrm{~m})=\tilde{\mathrm{A}}_{\mathrm{n}-\mathrm{m} n-\mathrm{m}}^{\mathrm{m}} .
\end{aligned}
$$


For $\mathrm{m} \in \mathrm{J}(0, \mathrm{n}-2)$, we denote

$$
\begin{aligned}
& \overline{\mathrm{D}}_{\mathrm{m}}=\operatorname{diag}\left(\epsilon_{1} \mathrm{I}_{1} \epsilon_{2} \mathrm{I}_{2} \ldots \epsilon_{\mathrm{n}-\mathrm{m}-1} \mathrm{I}_{\mathrm{n}-\mathrm{m}-1}\right) ; \\
& \hat{\mathrm{L}}_{\mathrm{m}}=-\hat{\mathrm{A}}_{22}(\mathrm{~m})^{-1} \hat{\mathrm{A}}_{21}(\mathrm{~m}) ; \\
& \hat{\mathrm{M}}_{\mathrm{m}}=\hat{\mathrm{A}}_{12}(\mathrm{~m}) \hat{\mathrm{A}}_{22}(\mathrm{~m})^{-1} .
\end{aligned}
$$

\section{Diagonalisation Process.}

In this section our aim is to develop a procedure to totally decouple the original system (2.5). This can be done by carrying over the procedure exactly in analogous manner as in [6]. The only difference is that the boundary conditions are changed in the process due to the application of transformation. By following a procedure analogous to section 4 in [6], at $m-$ th step, we will have,

$$
\left\{\begin{array}{l}
S_{n-m ~ c:} \quad \dot{X}_{n-m}^{m}=A^{m} X_{n-m}^{m} \\
S_{n-m d} \quad \epsilon_{n-i} \dot{X}_{n-i}(i+1)=\left(A_{22}(i)-\epsilon_{n-i} L_{i} A_{12}(i)\right) X_{n-i}(i+1), \text { for } i \in J(0, m-1) . \\
Q(m, \epsilon) X_{n m-1}^{m ~ 0}+R(m, \epsilon) X_{n m-1}^{m ~ 1}=B(\epsilon), \quad m \in J(0, n-2)
\end{array}\right.
$$

Coupled system $\left(4.1 \mathrm{~S}_{\mathrm{n}}-\mathrm{m} \mathrm{c}\right)$ can be rewritten as

$$
\begin{aligned}
& \left\{\begin{array}{c}
\dot{\mathrm{X}}_{\mathrm{n}-\mathrm{m}-1}^{\mathrm{m}}=\mathrm{A}_{11}(\mathrm{~m}) \mathrm{X}_{\mathrm{n}-\mathrm{m}-1}^{\mathrm{m}}+\mathrm{A}_{12}(\mathrm{~m}) \mathrm{X}_{\mathrm{n}-\mathrm{m}}(\mathrm{m}), \\
\epsilon_{\mathrm{n}-\mathrm{m}} \dot{\mathrm{X}}_{\mathrm{n}-\mathrm{m}}(\mathrm{m})=\mathrm{A}_{21}(\mathrm{~m}) \mathrm{X}_{\mathrm{n}-\mathrm{m}-1}^{\mathrm{m}}+\mathrm{A}_{22}(\mathrm{~m}) \mathrm{X}_{\mathrm{n}-\mathrm{m}}(\mathrm{m}), \\
\epsilon_{\mathrm{n}-\mathrm{i}} \dot{\mathrm{X}}_{\mathrm{n}-\mathrm{i}}(\mathrm{i}+1)=\left(\mathrm{A}_{22}(\mathrm{i})-\epsilon_{\mathrm{n}-\mathrm{i}} \mathrm{L}_{\mathrm{i}} \mathrm{A}_{12}(\mathrm{i})\right) \mathrm{X}_{\mathrm{n}-\mathrm{i}}(\mathrm{i}+1), \text { for } \mathrm{i} \in \mathrm{J}(0, \mathrm{~m}-1)
\end{array}\right. \\
& \mathrm{Q}(\mathrm{m}, \epsilon) \mathrm{X}_{\mathrm{nm}-1}^{\mathrm{m} \mathrm{0}}+\mathrm{R}(\mathrm{m}, \epsilon) \mathrm{X}_{\mathrm{nm}-1}^{\mathrm{m} 1}=\mathrm{B}(\epsilon)
\end{aligned}
$$

Assumption A 4.1: $\mathrm{A}_{22}(\mathrm{~m})$ is non-singular.

Now we apply Chang's transformation [1] defined by

$$
\mathrm{X}_{\mathrm{n}-\mathrm{m}}^{\mathrm{m}+1}=\tilde{\mathrm{T}}_{\mathrm{n}-\mathrm{m}} \mathrm{X}_{\mathrm{n}-\mathrm{m}}^{\mathrm{m}}
$$

to system (4.3) with (4.4),

where

$$
\tilde{\mathrm{T}}_{\mathrm{n}}=\mathrm{T}_{\mathrm{n}},
$$




$$
\begin{array}{ll}
\tilde{T}_{\mathrm{n}-\mathrm{m}} & =\left[\begin{array}{cc}
\mathrm{T}_{\mathrm{n}-\mathrm{m}} & 0 \\
0 & \mathrm{I}(\mathrm{n}-\mathrm{m})
\end{array}\right], \text { for } \mathrm{m} \in \mathrm{J}(1, \mathrm{n}-2) \\
\text { and } & \mathrm{T}_{\mathrm{n}-\mathrm{m}}=\left[\begin{array}{cc}
\mathrm{I}^{\mathrm{n}-\mathrm{m}-1}+\epsilon_{\mathrm{n}-\mathrm{m}} \mathrm{M}_{\mathrm{m}} \mathrm{L}_{\mathrm{m}}-\epsilon_{\mathrm{n}-\mathrm{m}} \mathrm{M}_{\mathrm{m}} \\
-\mathrm{L}_{\mathrm{m}}
\end{array}\right] \text {, for } \mathrm{m} \in \mathrm{J}(0, \mathrm{n}-2) .
\end{array}
$$

and $L_{m}, M_{m}$ satisfy the following differential equations:

$$
\left\{\begin{array}{l}
\epsilon_{\mathrm{n}-\mathrm{m}} \dot{\mathrm{L}}_{\mathrm{m}}=-\epsilon_{\mathrm{n}-\mathrm{m}} \mathrm{L}_{\mathrm{m}}\left(\mathrm{A}_{11}(\mathrm{~m})+\mathrm{A}_{12}(\mathrm{~m}) \mathrm{L}_{\mathrm{m}}\right)+\mathrm{A}_{21}(\mathrm{~m})+\mathrm{A}_{22}(\mathrm{~m}) \mathrm{L}_{\mathrm{m}}, \\
\epsilon_{\mathrm{n}-\mathrm{m}} \dot{\mathrm{M}}_{\mathrm{m}}=-\mathrm{M}_{\mathrm{m}}\left(\mathrm{A}_{22}(\mathrm{~m})-\epsilon_{\mathrm{n}-\mathrm{m}} \mathrm{L}_{\mathrm{m}} \mathrm{A}_{12}(\mathrm{~m})\right)+\epsilon_{\mathrm{n}-\mathrm{m}}\left(\mathrm{A}_{11}(\mathrm{~m})+\mathrm{A}_{12}(\mathrm{~m}) \mathrm{L}_{\mathrm{m}}\right) \mathrm{M}_{\mathrm{m}}+\mathrm{A}_{12}(\mathrm{~m}) .
\end{array}\right.
$$

Then we will have

$$
\left\{\begin{array}{c}
\dot{\mathrm{X}}_{\mathrm{n}-\mathrm{m}-1}^{\mathrm{m}+1}=\left(\mathrm{A}_{11}(\mathrm{~m})+\mathrm{A}_{12}(\mathrm{~m}) \mathrm{L}_{\mathrm{m}}\right) \mathrm{X}_{\mathrm{n}-\mathrm{m}-1}^{\mathrm{m}+1} \\
\epsilon_{\mathrm{n}-\mathrm{i}} \dot{\mathrm{X}}_{\mathrm{n}-\mathrm{i}}(\mathrm{i}+1)=\left(\mathrm{A}_{22}(\mathrm{i})-\epsilon_{\mathrm{n}-\mathrm{i}} \mathrm{L}_{\mathrm{i}} \mathrm{A}_{12}(\mathrm{i})\right) \mathrm{X}_{\mathrm{n}-\mathrm{i}}(\mathrm{i}+1), \text { for } \mathrm{i} \in \mathrm{J}(0, \mathrm{~m}) \\
\mathrm{Q}(\mathrm{m}+1, \epsilon) \mathrm{X}_{\mathrm{nm}}^{\mathrm{m}+10}+\mathrm{R}(\mathrm{m}+1, \epsilon) \mathrm{X}_{\mathrm{nm}}^{\mathrm{m}+11}=\mathrm{B}(\epsilon)
\end{array}\right.
$$

where $Q(m+1, \epsilon)=Q(m, \epsilon) \tilde{T}_{n-m}^{-1}, \quad R(m+1, \epsilon)=R(m, \epsilon) \tilde{T}_{n-m}^{-1}$

Furthermore, this fast and slow mode decoupling procedure terminates after $(n-1)$ steps. In the light of this, $m$ in (4.1) and (4.2) belongs to $J(1, n-1)$. At the end of $(n-1)$ steps the original system (2.4) with (2.5) will be complet-ly decoupled.

This totally decoupled system is rewritten as

$$
\left\{\begin{array}{c}
\dot{\mathrm{U}}_{1}=\mathrm{A}_{11}(\mathrm{n}-1) \mathrm{U}_{1}, \\
\epsilon_{\mathrm{n}-\mathrm{m}} \dot{\mathrm{U}}_{\mathrm{n}-\mathrm{m}}=\left[\mathrm{A}_{22}(\mathrm{~m})-\epsilon_{\mathrm{n}-\mathrm{m}} \mathrm{L}_{\mathrm{m}} \mathrm{A}_{12}(\mathrm{~m})\right] \mathrm{U}_{\mathrm{n}-\mathrm{m}}, \text { for } \mathrm{m} \in \mathrm{J}(0, \mathrm{n}-2) . \\
\mathrm{Q}(\mathrm{n}-1, \epsilon) \mathrm{U}^{0}+\mathrm{R}(\mathrm{n}-1, \epsilon) \mathrm{U}^{1}=\mathrm{B}(\epsilon),
\end{array}\right.
$$

Remark 4.1: From (4.7), for $\mathrm{m} \in \mathrm{J}(0, \mathrm{n}-2), \mathrm{T}_{\mathrm{n}-\mathrm{m}}$ is determined by the following differential equations. 


$$
\left\{\begin{aligned}
\epsilon_{\mathrm{n}-\mathrm{m}} \dot{\mathrm{L}}_{\mathrm{m}}= & -\epsilon_{\mathrm{n}-\mathrm{m}} \mathrm{L}_{\mathrm{m}}\left(\mathrm{A}_{11}(\mathrm{~m})+\mathrm{A}_{12}(\mathrm{~m}) \mathrm{L}_{\mathrm{m}}\right)+\mathrm{A}_{21}(\mathrm{~m})+\mathrm{A}_{22}(\mathrm{~m}) \mathrm{L}_{\mathrm{m}} \\
\epsilon_{\mathrm{n}-\mathrm{m}} \dot{\bar{M}}_{\mathrm{m}}= & -\overline{\mathrm{M}}_{\mathrm{m}}\left(\mathrm{A}_{22}(\mathrm{~m})-\epsilon_{\mathrm{n}-\mathrm{m}} \mathrm{L}_{\mathrm{m}} \mathrm{A}_{12}(\mathrm{~m})\right)+\epsilon_{\mathrm{n}-\mathrm{m}} \overline{\mathrm{D}}_{\mathrm{m}}\left(\mathrm{A}_{11}(\mathrm{~m})+\mathrm{A}_{12}(\mathrm{~m}) \mathrm{L}_{\mathrm{m}}\right) \overline{\mathrm{D}}_{\mathrm{m}}^{-1} \overline{\mathrm{M}}_{\mathrm{m}} \\
& +\overline{\mathrm{D}}_{\mathrm{m}} \mathrm{A}_{12}(\mathrm{~m})
\end{aligned}\right.
$$

Where $\bar{M}_{m}=\bar{D}_{m} M_{m}$.

Remark 4.2: We remark that instead of applying transformations $\tilde{\mathrm{T}}_{\mathrm{n}}, \tilde{\mathrm{T}}_{\mathrm{n}-1}, \ldots, \tilde{\mathrm{T}}_{\mathrm{n}-\mathrm{m}}$ successively, to the original system $(m+1)$ times, we can apply a composition of transformation

$$
\mathrm{T}^{\mathrm{n}-\mathrm{m}}=\tilde{\mathrm{T}}_{\mathrm{n}-\mathrm{m}} \mathrm{o} \tilde{\mathrm{T}}_{\mathrm{n}-\mathrm{m}+1} \mathrm{o} \ldots . \tilde{\mathrm{T}}_{\mathrm{n}}
$$

to the original system (2.5). An application of this composite transformation $\mathrm{T}^{\mathrm{n}-\mathrm{m}}$ to (2.5) gives rise to (4.8). The structure of $\mathrm{T}^{\mathrm{n}-\mathrm{m}}$ is as follows.

$$
\begin{aligned}
& \mathrm{T}^{\mathrm{n}-\mathrm{m}}=\left[\begin{array}{cc}
\mathrm{T}_{\mathrm{n}-\mathrm{m}} & 0 \\
0 & \mathrm{I}(\mathrm{n}-\mathrm{m})
\end{array}\right] \mathrm{o}\left[\begin{array}{cc}
\mathrm{P}_{\mathrm{n}-\mathrm{m}+1^{(1)}}^{1} & \mathrm{C}_{\mathrm{n}-\mathrm{m}+1^{(1)}} \\
\mathrm{R}_{\mathrm{n}-\mathrm{m}+1^{(1)}} & \mathrm{P}_{\mathrm{n}-\mathrm{m}+1^{(1)}}^{2}
\end{array}\right] \mathrm{o}\left[\begin{array}{cc}
\mathrm{P}_{\mathrm{n}-\mathrm{m}+2^{(2)}}^{1} & \mathrm{C}_{\mathrm{n}-\mathrm{m}+2^{(2)}} \\
\mathrm{R}_{\mathrm{n}-\mathrm{m}+2^{(2)}} & \mathrm{P}_{\mathrm{n}-\mathrm{m}+2^{(2)}}^{2}
\end{array}\right] 0 \\
& \circ \ldots \circ\left[\begin{array}{ll}
P_{n}^{1}(m) & C_{n}(m) \\
R_{n}(m) & P_{n}^{2}(m)
\end{array}\right]
\end{aligned}
$$

where, for all $m \in J(0, n-2)$ and $q \in J(0, m-1), P_{n-q}^{1}(k), P_{n-q}^{2}(k), C_{n-q}(k), R_{n-q}(k)$ are given by

for $1 \leq k \leq n-q-1$,

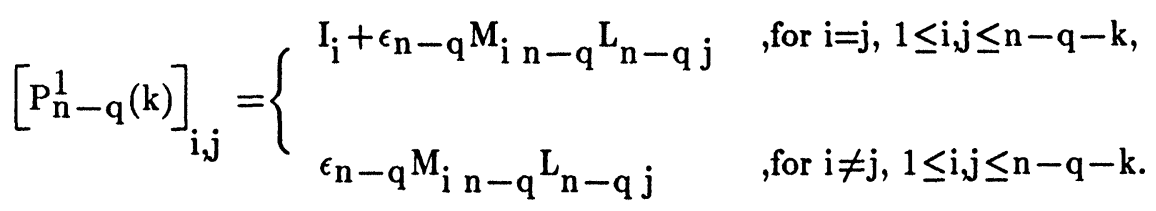

$$
\begin{aligned}
& {\left[R_{n}(1)\right]_{i, j}=-L_{n j}, \text { for } i=1,1 \leq j \leq n-q-1} \\
& {\left[R_{n-q}(1)\right]_{i, j}= \begin{cases}-L_{n-q j} & , \text { for } i=1,1 \leq j \leq n-q-1, \\
0 & , \text { for } 2 \leq i \leq q+1,1 \leq j \leq n-q-1\end{cases} }
\end{aligned}
$$


for $2 \leq k \leq n-q-1$

$$
\begin{aligned}
& {\left[R_{n-q}(k)\right]_{i, j}= \begin{cases}\epsilon_{n-q} M_{n-q-k+i ~ n-q} L_{n-q j} & , \text { for } i=j, 1 \leq i, j \leq n-q-k \\
{\left[R_{n-q}(k-1)\right]_{i, j}} & , \text { for } 2 \leq i \leq k+q, 1 \leq j \leq n-q\end{cases} } \\
& {\left[C_{n}(1)\right]_{i, 1}=-\epsilon_{n} M_{i n}, \text { for } 1 \leq i \leq n-q-1,} \\
& {\left[C_{n-q}(1)\right]_{i, j}= \begin{cases}-\epsilon_{n-q} M_{i n-q} & , \text { for } 1 \leq i \leq n-q-1, j=1, \\
0 & , \text { for } 1 \leq i \leq n-q+1,2 \leq j \leq q+1 .\end{cases} }
\end{aligned}
$$

for $2 \leq k \leq n-q-1$

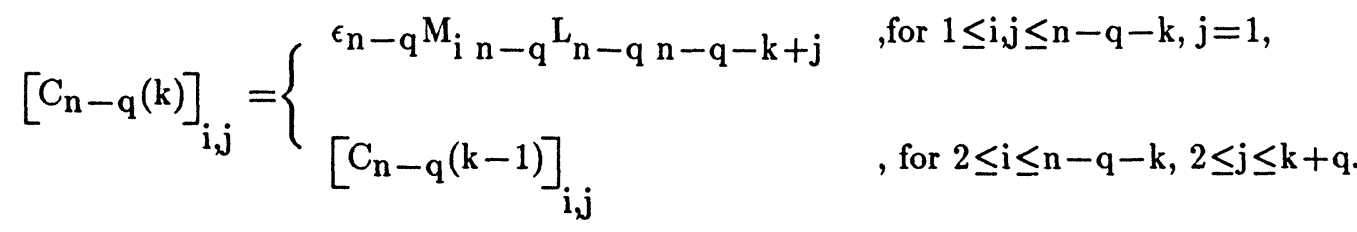

$$
\begin{aligned}
& \mathrm{P}_{\mathrm{n}}^{2}(1)=\mathrm{I}_{\mathrm{n}}, \\
& P_{n-q}^{2}(1)=I(n-q) \text {, } \\
& {\left[P_{n}^{2}(2)\right]_{i j}=\left[\begin{array}{lr}
I_{n-1}+\epsilon_{n} M_{n-1} L_{n n-1}, & \text {,for } i=1, j=1 \\
-L_{n n-1} & , \text { for } i=2, j=1 \\
-\epsilon_{n} M_{n-1 n} & , \text { for } i=1, j=2 \\
I_{n} & , \text { for } i=2, j=2
\end{array}\right.} \\
& {\left[P_{n-q}^{2}(2)\right]_{i j}=\left[\begin{array}{lr}
I_{n-q}+\epsilon_{n-q} M_{n-q-1} n-q & L_{n-q n-q-1} \\
-L_{n-q n}-q-1 & , \text { for } i=1, j=1, \\
-\epsilon_{n-q} M_{n-q-1 n-q} & , \text { for } i=1, j=2, j=1, \\
{\left[P_{n-q}^{2}(1)\right]_{i j}} & \text {,for } i=2, j=2, \\
0, \text { for } 3 \leq i \leq q+2, j=1 \text { and } i=1,3 \leq j \leq q+2, &
\end{array}\right.}
\end{aligned}
$$




$$
\begin{aligned}
& {\left[P_{n}^{2}(k)\right]_{i j}=\left[\begin{array}{lr}
I_{n-k+1}+\epsilon_{n} M_{n-k+1} L_{n n-k+1} & , \text { for } i=1, j=1, \\
\epsilon_{n} M_{n-k+i n} L_{n n-k+j} & \text {,for } 2 \leq i \leq k-1, j=1, \text { and } i=1,2 \leq j \leq k-1, \\
-L_{n n-k+1} & , \text { for } i=k, j=1, \\
-\epsilon_{n} M_{n-k+1 n} & \text {,for } i=1, j=k, \\
{\left[P_{n}^{2}(k-1)\right]_{i j}} & \text {,for } 2 \leq i, j \leq k,
\end{array}\right.}
\end{aligned}
$$

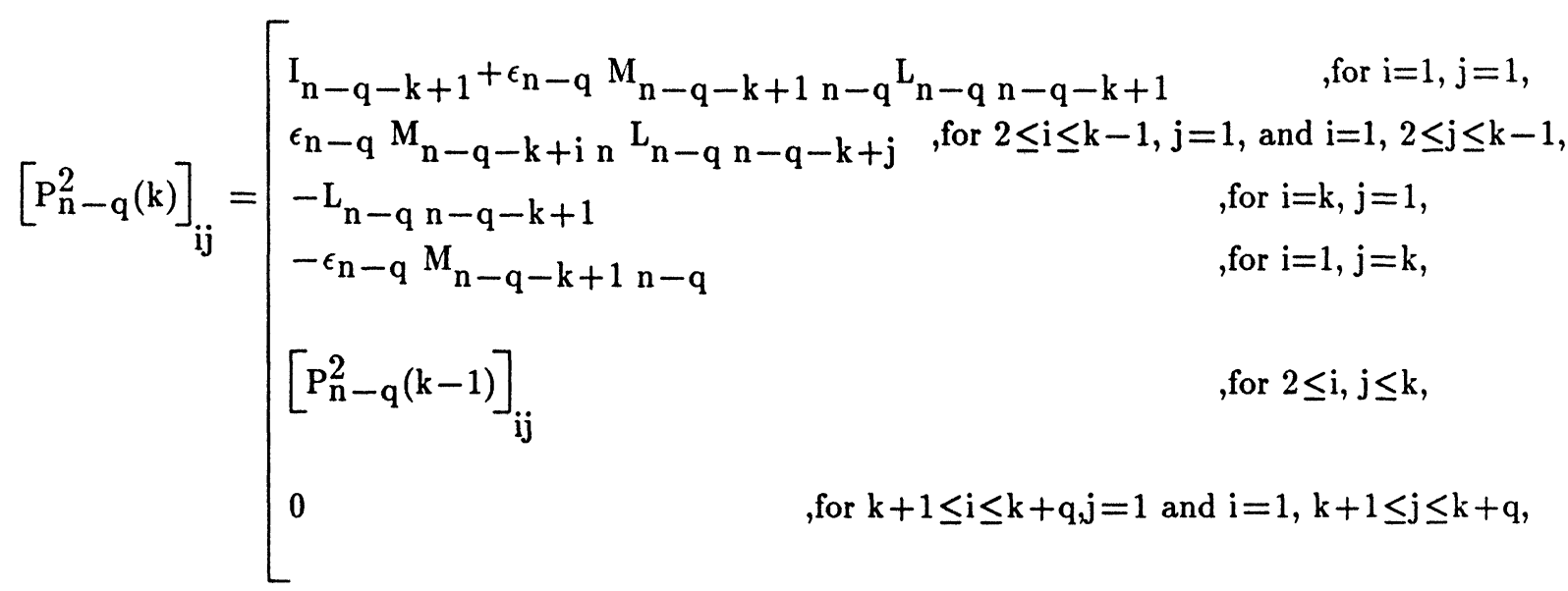

We note that $\mathrm{T}^{2}$, transforms the original system $(2.5)-(2.6)$ into totally decoupled system (4.10)-(4.11). Furthermore, we observe that

$$
\mathrm{S}_{\mathrm{n}-\mathrm{m}}=\left[\begin{array}{cc}
\mathrm{I}^{\mathrm{n}-\mathrm{m}-1} & \epsilon_{\mathrm{n}-\mathrm{m}} \mathrm{M}_{\mathrm{m}} \\
\mathrm{L}_{\mathrm{m}} & \mathrm{I}_{\mathrm{n}-\mathrm{m}}+\epsilon_{\mathrm{n}-\mathrm{m}} \mathrm{L}_{\mathrm{m}} \mathrm{M}_{\mathrm{m}}
\end{array}\right]
$$

is the inverse of $T_{n-m}$, for $m \in J(0, n-2)$, and hence $\tilde{T}_{n-m}$ is invertible. Denoting $\tilde{S}_{n-m}$ as the inverse of $\tilde{\mathrm{T}}_{\mathrm{n}-\mathrm{m}}, \mathrm{S}^{2}=\tilde{\mathrm{S}}_{\mathrm{n}} \circ \tilde{\mathrm{S}}_{\mathrm{n}-1} \mathrm{o \ldots o} \tilde{\mathrm{S}}_{2}$ is an inverse of $\mathrm{T}^{2}$. Therefore the solution of the original system can be given interms of the solution of the totally decoupled multi time scale system $(4.10)-(4.11)$ by

$$
X(t)=S^{2}(t) U(t)
$$

where $U(t)$ is the slution of $(4.10)-(4.11)$.

The validity of the transformation is established, by establishing the existence, uniqueness, boundedness and other fundamental properties of (4.7) for $m \in J(0, n-2)$ in [7]. For the sake of completeness, we present the desired assumptions. 
Assumption A4.1: For every $\mathrm{m} \in \mathrm{J}(0, \mathrm{n}-2)$, the absolute value of the real part of all eigen values of $\hat{\mathrm{A}}_{22}(\mathrm{~m})$ are greater than or equal to $2 \alpha_{\mathrm{m}}$ where $\alpha_{\mathrm{m}}>0$.

Assumption A4.2: Coefficient matrix functions $A_{i j}, i, j \in J(1, n)$ in system $(2.4)$ and $\hat{A}_{22}^{-1}(m)$, $\mathrm{m} \in \mathrm{J}(0, \mathrm{n}-2)$ are bounded.

Assumption $\mathrm{A} 4.3: \quad \hat{\mathrm{A}}_{22}^{-1}(\mathrm{~m}) \hat{\mathrm{A}}_{21}(\mathrm{~m}), \hat{\mathrm{A}}_{12}(\mathrm{~m}) \hat{\mathrm{A}}_{22}^{-1}(\mathrm{~m}), \mathrm{m} \in \mathrm{J}(0, \mathrm{n}-2)$, are bounded and satisfies the following lipschitz condition.

$$
\begin{aligned}
& \left\|\hat{\mathrm{A}}_{22}^{-1}(\mathrm{~m}, \mathrm{t}) \hat{\mathrm{A}}_{21}(\mathrm{~m}, \mathrm{t})-\hat{\mathrm{A}}_{22}^{-1}(\mathrm{~m}, \mathrm{~s}) \hat{\mathrm{A}}_{21}(\mathrm{~m}, \mathrm{~s})\right\| \leq \mathrm{l}_{\mathrm{m}}|\mathrm{t}-\mathrm{s}| \text { for } 0 \leq \mathrm{t}, \mathrm{s} \leq 1, \\
& \left\|\hat{\mathrm{A}}_{12}(\mathrm{~m}, \mathrm{t}) \hat{\mathrm{A}}_{22}^{-1}(\mathrm{~m}, \mathrm{t})-\hat{\mathrm{A}}_{12}(\mathrm{~m}, \mathrm{~s}) \hat{\mathrm{A}}_{22}^{-1}(\mathrm{~m}, \mathrm{~s})\right\| \leq \mathrm{m}_{\mathrm{m}}|\mathrm{t}-\mathrm{s}| \text { for } 0 \leq \mathrm{t}, \mathrm{s} \leq 1,
\end{aligned}
$$

for some positive real numbers $\mathrm{l}_{\mathrm{m}}$ and $\mathrm{m}_{\mathrm{m}}$.

Remark 4.3: From the boundedness of coefficient matrices $A_{i, j}, i, j \in J(1, n)$, and the definition of $A_{11}(0), A_{12}(0), A_{21}(0)$ and $A_{22}(0)$, it is clear that $\epsilon_{n} A_{11}(0), \epsilon_{n} A_{12}(0), A_{21}(0)$ and $A_{22}(0)$ are bounded. If $L_{0}$ is bounded then it is clear that $\epsilon_{n-1} A_{11}(1), \epsilon_{n-1} A_{12}(1), A_{21}(1)$ and $A_{22}(1)$ are bounded. Continuing this process, one can conclude that if $\mathrm{L}_{\mathrm{m}-1}$ is bounded, then it follows that $\epsilon_{\mathrm{n}-\mathrm{m}} \mathrm{A}_{11}(\mathrm{~m}), \epsilon_{\mathrm{n}-\mathrm{m}} \mathrm{A}_{12}(\mathrm{~m}), \mathrm{A}_{21}(\mathrm{~m})$ and $\mathrm{A}_{22}(\mathrm{~m})$ are bounded.

$$
\begin{aligned}
& \text { Moreover, if } \mathrm{L}_{0}=\hat{\mathrm{L}}_{0}+\mathrm{O}\left(\frac{\epsilon_{\mathrm{n}}}{\epsilon_{\mathrm{n}-1}}\right) \text { then } \\
& \mathrm{A}_{22}(1)=\hat{\mathrm{A}}_{22}(1)+\mathrm{O}\left(\frac{\epsilon_{\mathrm{n}}}{\epsilon_{\mathrm{n}-1}}\right), \mathrm{A}_{21}(1)=\hat{\mathrm{A}}_{21}(1)+\mathrm{O}\left(\frac{\epsilon_{\mathrm{n}}}{\epsilon_{\mathrm{n}-1}}\right), \\
& \mathrm{A}_{12}(1)=\hat{\mathrm{A}}_{12}(1)+\mathrm{D}_{1} \mathrm{O}\left(\frac{\epsilon_{\mathrm{n}}}{\epsilon_{\mathrm{n}-1}}\right), \mathrm{A}_{11}(1)=\hat{\mathrm{A}}_{11}(1)+\mathrm{D}_{1} \mathrm{O}\left(\frac{\epsilon_{\mathrm{n}}}{\epsilon_{\mathrm{n}-1}}\right) . \text { Continuing in }
\end{aligned}
$$

this manner, one can conclude that if $\mathrm{L}_{\mathrm{m}}=\hat{\mathrm{L}}_{\mathrm{m}}+\sum_{\mathrm{k}=\mathrm{n}-\mathrm{m}}^{\mathrm{n}} \mathrm{O}\left(\frac{{ }^{\epsilon} \mathrm{k}}{\epsilon_{\mathrm{k}-1}}\right)$, then

$$
\begin{aligned}
& \mathrm{A}_{22}(\mathrm{~m}+1)=\hat{\mathrm{A}}_{22}(\mathrm{~m}+1)+\sum_{\mathrm{k}=\mathrm{n}-\mathrm{m}}^{\mathrm{n}} \mathrm{O}\left(\frac{\epsilon_{\mathrm{k}}}{\epsilon \mathrm{k}-1}\right), \mathrm{A}_{21}(\mathrm{~m}+1)=\hat{\mathrm{A}}_{21}(\mathrm{~m}+1)+\sum_{\mathrm{k}=\mathrm{n}-\mathrm{m}}^{\mathrm{n}} \mathrm{O}\left(\frac{\epsilon_{\mathrm{k}}}{\epsilon_{\mathrm{k}-1}}\right), \\
& \mathrm{A}_{12}(\mathrm{~m}+1)=\hat{\mathrm{A}}_{12}(\mathrm{~m}+1)+\mathrm{D}_{\mathrm{m}+\mathrm{k}=\mathrm{n}-\mathrm{m}} \sum^{\mathrm{n}} \mathrm{O}\left(\frac{\epsilon_{\mathrm{k}}}{\epsilon_{\mathrm{k}-1}}\right), \mathrm{A}_{11}(\mathrm{~m}+1)=\hat{\mathrm{A}}_{11}(\mathrm{~m}+1)+\mathrm{D}_{\mathrm{m}}+1 \sum_{\mathrm{k}=\mathrm{n}-\mathrm{m}}^{\mathrm{n}} \mathrm{O}\left(\frac{\epsilon_{\mathrm{k}}}{\epsilon_{\mathrm{k}-1}}\right) .
\end{aligned}
$$

Remark 4.4: From the approximations of $\mathrm{L}_{\mathrm{m}}$ and $\mathrm{M}_{\mathrm{m}}$, it can be observed that 
where

$$
\mathrm{T}^{2}=\hat{\mathrm{T}}^{2}+\sum_{\mathrm{k}=2}^{\mathrm{n}} \mathrm{O}\left(\frac{\epsilon_{\mathrm{k}}}{\epsilon \mathrm{k}-1}\right)
$$

$$
\hat{\mathrm{T}}^{2}=\hat{\mathrm{T}}_{2} \mathrm{o} \ldots \mathrm{o} \hat{\mathrm{T}}_{\mathrm{n}}
$$

and

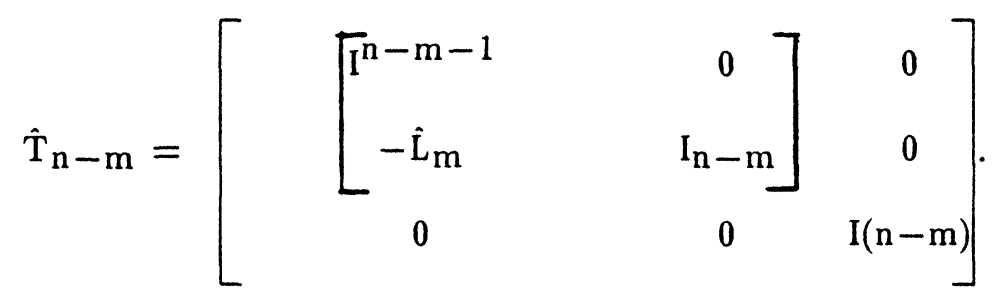

Similarly

$$
\mathrm{S}^{2}=\hat{\mathrm{S}}^{2}+\sum_{\mathrm{k}=2}^{\mathrm{n}} \mathrm{O}\left(\frac{\epsilon \mathrm{k}}{\epsilon_{\mathrm{k}-1}}\right)
$$

where

$$
\hat{\mathrm{S}}^{2}=\hat{\mathrm{S}}_{\mathrm{n}} \circ \ldots \mathrm{os}_{2}
$$

and

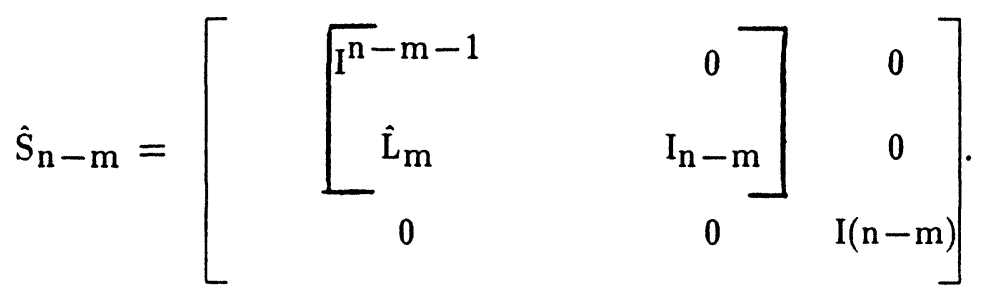

\section{Existence and approximation.}

In this section we give sufficient conditions to establish the existence of solution and to find an approximate solution of the original problem (2.5)-(2.6). The obtained conditions are easy to verify and computationally feasible. This is accomplished by developing a dichotomy transformation which decompose the faster sub-systems in the totally decoupled system (4.10) into stable and unstable subsystems.

For this purpose let us introduce a few notations.

For $\mathrm{m} \in \mathrm{J}(0, \mathrm{n}-2)$,

$$
A_{22}(m)-\epsilon_{n-m} L_{m} A_{12}(m)=\left[\begin{array}{cc}
F_{11}(m) & F_{12}(m) \\
F_{21}(m) & F_{22}(m)
\end{array}\right]
$$

where $F_{11}(\mathrm{~m}), \mathrm{F}_{12}(\mathrm{~m}), \mathrm{F}_{21}(\mathrm{~m})$ and $\mathrm{F}_{22}(\mathrm{~m})$ are of dimensions $\mathrm{k}_{\mathrm{m}} \mathrm{xk}_{\mathrm{m}}, \mathrm{k}_{\mathrm{m}} \mathrm{x}\left(\mathrm{n}_{\mathrm{n}-\mathrm{m}}-\mathrm{k}_{\mathrm{m}}\right)$, 
$\left(n_{n}-m-k_{m}\right) \times k_{m}$ and $\left(n_{n}-m-k_{m}\right) x\left(n_{n}-m-k_{m}\right)$ respectively. $k_{m}$ 's are number of eigenvalues of $\hat{\mathrm{A}}_{22}(\mathrm{~m})$ with negative real part.

$$
\hat{A}_{22}(\mathrm{~m})=\left[\begin{array}{cc}
\hat{\mathrm{F}}_{11}(\mathrm{~m}) & \hat{\mathrm{F}}_{12}(\mathrm{~m}) \\
\hat{\mathrm{F}}_{21}(\mathrm{~m}) & \hat{\mathrm{F}}_{22}(\mathrm{~m})
\end{array}\right]
$$

where $\hat{F}_{i j}(m)$ 's have the same dimensions as of $F_{i j}(m)$ 's for $i, j \in J(1,2)$.

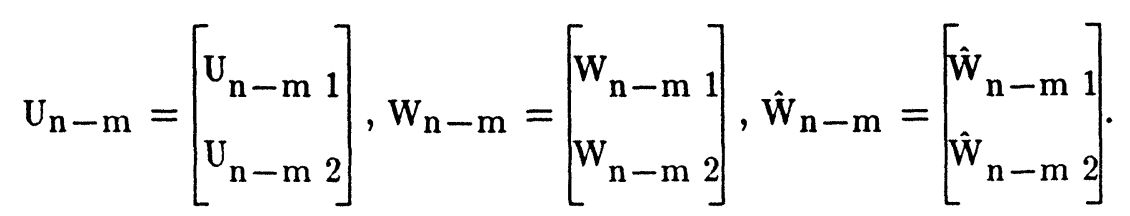

where $U_{n-m 1}, W_{n-m 1}, \hat{W}_{n-m 1}$ are of dimensions $k_{m} \times 1$ and $U_{n-m ~ 2}, W_{n-m 2}, \hat{W}_{n-m 2}$ are of dimensions $\left(\mathrm{n}_{\mathrm{n}}-\mathrm{m}-\mathrm{k}_{\mathrm{m}}\right) \mathrm{x} 1$.

Now we consider the totally decoupled system (4.10). Faster sub-systems in (4.10) can be rewritten as

$$
\epsilon_{\mathrm{n}-\mathrm{m}}\left[\begin{array}{c}
\dot{\mathrm{U}}_{\mathrm{n}-\mathrm{m} 1} \\
\dot{\mathrm{U}}_{\mathrm{n}-\mathrm{m} 2}
\end{array}\right]=\left[\begin{array}{cc}
\mathrm{F}_{11}(\mathrm{~m}) & \mathrm{F}_{12}(\mathrm{~m}) \\
\mathrm{F}_{21}(\mathrm{~m}) & \mathrm{F}_{22}(\mathrm{~m})
\end{array}\right]\left[\begin{array}{c}
\mathrm{U}_{\mathrm{n}-\mathrm{m} 1} \\
\mathrm{U}_{\mathrm{n}-\mathrm{m} 2}
\end{array}\right], \mathrm{m} \in \mathrm{J}(0, \mathrm{n}-2) .
$$

We apply a transformation defined by

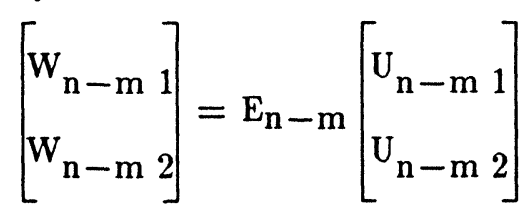

to system (5.1),

where

$$
E_{n-m}=\left[\begin{array}{cc}
I_{k_{m}}+P_{m} N_{m} & -P_{m} \\
-N_{m} & I_{n_{n}-m}-k_{m}
\end{array}\right]
$$

and $\mathrm{N}_{\mathrm{m}}, \mathrm{P}_{\mathrm{m}}$ satisfy the following diferential equations

$$
\begin{aligned}
& \left\{\begin{array}{l}
\epsilon_{\mathrm{n}-\mathrm{m}} \dot{\mathrm{N}}_{\mathrm{m}}=-\mathrm{N}_{\mathrm{m}}\left(\mathrm{F}_{11}(\mathrm{~m})+\mathrm{F}_{12}(\mathrm{~m}) \mathrm{N}_{\mathrm{m}}\right)+\mathrm{F}_{21}(\mathrm{~m})+\mathrm{F}_{22}(\mathrm{~m}) \mathrm{N}_{\mathrm{m}}, \\
\mathrm{N}_{\mathrm{m}}(0)=\mathrm{N}_{\mathrm{m}}^{0},
\end{array}\right. \\
& \left\{\begin{array}{l}
\epsilon_{\mathrm{n}-\mathrm{m}} \dot{\mathrm{P}}_{\mathrm{m}}=-\mathrm{P}_{\mathrm{m}}\left(\mathrm{F}_{22}(\mathrm{~m})-\mathrm{N}_{\mathrm{m}} \mathrm{F}_{12}(\mathrm{~m})\right)+\left(\mathrm{F}_{11}(\mathrm{~m})+\mathrm{F}_{12}(\mathrm{~m}) \mathrm{N}_{\mathrm{m}}\right) \mathrm{P}_{\mathrm{m}}+\mathrm{F}_{12}(\mathrm{~m}) \\
\mathrm{P}_{\mathrm{m}}(1)=\mathrm{P}_{\mathrm{m}}^{1} .
\end{array}\right.
\end{aligned}
$$


Then we will have

$$
\epsilon_{\mathrm{n}-\mathrm{m}}\left[\begin{array}{l}
\dot{\mathrm{W}}_{\mathrm{n}-\mathrm{m} \mathrm{1}} \\
\dot{\mathrm{W}}_{\mathrm{n}-\mathrm{m} 2}
\end{array}\right]=\left[\begin{array}{cc}
\mathrm{F}_{11}(\mathrm{~m})+\mathrm{F}_{12}(\mathrm{~m}) \mathrm{N}_{\mathrm{m}} & 0 \\
0 & \mathrm{~F}_{22}(\mathrm{~m})-\mathrm{N}_{\mathrm{m}} \mathrm{F}_{12}(\mathrm{~m})
\end{array}\right]\left[\begin{array}{l}
\mathrm{W}_{\mathrm{n}-\mathrm{m} 1} \\
\mathrm{~W}_{\mathrm{n}-\mathrm{m} 2}
\end{array}\right] .
$$

For the validity of the transformation $\mathrm{E}_{\mathrm{n}-\mathrm{m}}$, we will consider the algebraic equation corresponding to $(5.2)$

$$
0=-\bar{N}_{m} \hat{\mathrm{F}}_{11}(\mathrm{~m})+\hat{\mathrm{F}}_{22}(\mathrm{~m}) \overline{\mathrm{N}}_{\mathrm{m}}-\overline{\mathrm{N}}_{\mathrm{m}} \hat{\mathrm{F}}_{12}(\mathrm{~m}) \overline{\mathrm{N}}_{\mathrm{m}}+\overline{\mathrm{F}}_{21}(\mathrm{~m})
$$

and assume the following assumption for a solution $\overline{\mathrm{N}}_{\mathrm{m}}(\mathrm{t})$ of $(5.5)$ for $0 \leq \mathrm{t} \leq 1$.

Assumption A5.1: For $m \in J(0, n-2)$ equation (5.5) has a bounded solution $\overline{\mathrm{N}}_{\mathrm{m}}(\mathrm{t}), \mathrm{t} \in[0,1]$ and it has a bounded derivative for every $t \in[0,1]$. Furthermore, assume that $\hat{\mathrm{F}}_{11}(\mathrm{~m})+\hat{\mathrm{F}}_{12}(\mathrm{~m}) \overline{\mathrm{N}}_{\mathrm{m}}$ and $\hat{\mathrm{F}}_{22}(\mathrm{~m})-\overline{\mathrm{N}}_{\mathrm{m}} \hat{\mathrm{F}}_{12}(\mathrm{~m})$ are unstable and stable matrices, respectively.

Theorem 5.1: Let the assumptions A2.1, A4.1, A4.2, A4.3 and A5.1 be satisfied. Then equation (5.2) has a solution $\mathrm{N}_{\mathrm{m}}$ and

$$
\mathrm{N}_{\mathrm{m}}=\overline{\mathrm{N}}_{\mathrm{m}}+\sum_{\mathrm{k}=\mathrm{n}-\mathrm{m}}^{\mathrm{n}} \mathrm{O}\left(\frac{\epsilon_{\mathrm{k}}}{\epsilon_{\mathrm{k}-1}}\right)
$$

where $\overline{\mathrm{N}}_{\mathrm{m}}$ is as in A5.1.

Moreover, $\quad \mathrm{F}_{11}(\mathrm{~m})+\mathrm{F}_{12}(\mathrm{~m}) \mathrm{N}_{\mathrm{m}}$ and $\mathrm{F}_{22}(\mathrm{~m})-\mathrm{N}_{\mathrm{m}} \mathrm{F}_{12}(\mathrm{~m})$ are unstable and stable matrices, respectively.

Proof: Let us consider the following differential equation

$$
\begin{aligned}
\epsilon_{\mathrm{n}-\mathrm{m}} \Delta \dot{\mathrm{P}}_{\mathrm{m}} & =-\Delta \mathrm{N}_{\mathrm{m}}\left(\mathrm{F}_{11}(\mathrm{~m})+\mathrm{F}_{12}(\mathrm{~m}) \overline{\mathrm{N}}_{\mathrm{m}}\right)+\left(\mathrm{F}_{22}(\mathrm{~m})-\overline{\mathrm{N}}_{\mathrm{m}} \mathrm{F}_{12}(\mathrm{~m})\right) \Delta \mathrm{N}_{\mathrm{m}}-\Delta \mathrm{N}_{\mathrm{m}} \mathrm{F}_{12}(\mathrm{~m}) \Delta \mathrm{N}_{\mathrm{m}}+\mathrm{l}_{\mathrm{m}} \\
\Delta \mathrm{N}_{\mathrm{m}}(0) & =\mathrm{N}_{\mathrm{m}}(0)-\overline{\mathrm{N}}_{\mathrm{m}}(0)
\end{aligned}
$$

where

$$
\mathrm{l}_{\mathrm{m}}=-\overline{\mathrm{N}}_{\mathrm{m}}\left(\mathrm{O}_{\mathrm{m}}+\mathrm{O}_{\mathrm{m}} \overline{\mathrm{N}}_{\mathrm{m}}\right)+\mathrm{O}_{\mathrm{m}} \overline{\mathrm{N}}_{\mathrm{m}}+\mathrm{O}_{\mathrm{m}}-\epsilon_{\mathrm{n}-\mathrm{m}} \dot{\overline{\mathrm{N}}}_{\mathrm{m}}
$$

and

$$
\mathrm{O}_{\mathrm{m}}=\sum_{\mathrm{k}=\mathrm{n}-\mathrm{m}}^{\mathrm{n}} \mathrm{O}\left(\frac{\epsilon_{\mathrm{k}}}{\epsilon_{\mathrm{k}-1}}\right)
$$

and we will show that

$$
\left\|\Delta N_{m}\right\| \leq \sum_{k=n-m}^{n} O\left(\frac{\epsilon_{k}}{\epsilon-1}\right)
$$


and

$$
\mathrm{N}_{\mathrm{m}}=\overline{\mathrm{N}}_{\mathrm{m}}+\Delta \mathrm{N}_{\mathrm{m}}
$$

We note that (5.7) has a unique solution.

Let $\phi_{1}(\mathrm{t}, \mathrm{s})$ and $\phi_{2}(\mathrm{t}, \mathrm{s})$ are fundamental matrix solutions of

$$
\begin{aligned}
& \epsilon_{\mathrm{n}-\mathrm{m}} \dot{\mathrm{Z}}=\left(\mathrm{F}_{11}(\mathrm{~m})+\mathrm{F}_{12}(\mathrm{~m}) \overline{\mathrm{N}}_{\mathrm{m}}\right) \mathrm{Z} \\
& \epsilon_{\mathrm{n}-\mathrm{m}} \dot{\mathrm{Y}}=\left(\mathrm{F}_{22}(\mathrm{~m})-\overline{\mathrm{N}}_{\mathrm{m}} \mathrm{F}_{12}(\mathrm{~m})\right) \mathrm{Y}, \text {, respectively. }
\end{aligned}
$$

From assumption A5.1, the approximation of $\mathrm{F}_{\mathrm{ij}}(\mathrm{m})$ and Lemma1 in [4], we have

$$
\begin{cases}\left\|\phi_{1}(\mathrm{t}, \mathrm{s})\right\| \leq \mathrm{K}_{1} \exp \left[-\frac{\alpha_{1}}{\epsilon_{\mathrm{n}-\mathrm{m}}}(\mathrm{s}-\mathrm{t})\right], & 0 \leq \mathrm{t} \leq \mathrm{s} \leq 1, \\ \left\|\phi_{2}(\mathrm{t}, \mathrm{s})\right\| \leq \mathrm{K}_{2} \exp \left[-\frac{\alpha_{2}}{\epsilon_{\mathrm{n}-\mathrm{m}}}(\mathrm{t}-\mathrm{s})\right], & 0 \leq \mathrm{s} \leq \mathrm{t} \leq 1\end{cases}
$$

where $\mathrm{K}_{1}, \mathrm{~K}_{2}, \alpha_{1}$ and $\alpha_{2}$ are some positive numbers independent of $\epsilon_{\mathrm{n}}-\mathrm{m}$.

A solution of (5.7) can be expressed as follows.

$\Delta \mathrm{N}_{\mathrm{m}}=\phi_{2}(\mathrm{t}, 0) \Delta \mathrm{N}_{\mathrm{m}}(0) \phi_{1}(0, \mathrm{t})+\int_{0}^{\mathrm{t}} \phi_{2}(\mathrm{t}, \mathrm{s}) \frac{1}{\epsilon_{\mathrm{n}-\mathrm{m}}}\left[\Delta \mathrm{N}_{\mathrm{m}}(\mathrm{s}) \mathrm{F}_{12}(\mathrm{~m})(\mathrm{s}) \Delta \mathrm{N}_{\mathrm{m}}(\mathrm{s})+\mathrm{l}_{\mathrm{m}}(\mathrm{s})\right] \phi_{1}(\mathrm{~s}, \mathrm{t}) \mathrm{ds}$

Now we will show that if

$$
\left\|\Delta \mathrm{N}_{\mathrm{m}}(0)\right\| \leq \rho_{1}
$$

then

$$
\left\|\Delta \mathrm{N}_{\mathrm{m}}(\mathrm{t})\right\| \leq \rho, \text { for all } 0 \leq \mathrm{t} \leq 1
$$

where $\rho, \rho_{1}$ will be defined later in this proof.

From (5.8) and (5.9), we have

$$
\left\|\Delta \mathrm{N}_{\mathrm{m}}(\mathrm{t})\right\| \leq \mathrm{K} \rho_{1} \exp \left[-\frac{\alpha}{\epsilon_{\mathrm{n}}-\mathrm{m}} \mathrm{t}\right]+\int_{0}^{\mathrm{t}} \mathrm{K} \exp \left[-\frac{\alpha}{\epsilon_{\mathrm{n}-\mathrm{m}}}(\mathrm{t}-\mathrm{s})\right]_{\frac{1}{\epsilon_{\mathrm{n}-\mathrm{m}}}}\left[\left\|\Delta \mathrm{N}_{\mathrm{m}}\right\| \rho \mathrm{k}_{12}+\mathrm{kO} \mathrm{O}\right] \mathrm{ds}
$$

where $\mathrm{K}=\mathrm{k}_{1}+\mathrm{k}_{2}, \alpha=\alpha_{1}+\alpha_{2},\left\|\mathrm{~F}_{12}\right\| \leq \mathrm{k}_{12}$ and $\mathrm{k}=2\left\|\overline{\mathrm{N}}_{\mathrm{m}}\right\|+\left\|\overline{\mathrm{N}}_{\mathrm{m}}\right\|^{2}+\left\|\overline{\mathrm{P}}_{\mathrm{m}}\right\|$.

set

$$
\mathrm{m}(\mathrm{t})=\exp \left[\frac{\alpha}{\epsilon_{\mathrm{n}}-\mathrm{m}} \mathrm{t}\right]\left\|\Delta \mathrm{N}_{\mathrm{m}}(\mathrm{t})\right\|
$$

From (5.10) and (5.11) we will have 


$$
\mathrm{m}(\mathrm{t}) \leq \mathrm{K} \rho_{1}+\int_{0}^{\mathrm{t}} \frac{1}{\epsilon_{\mathrm{n}-\mathrm{m}}}\left[\mathrm{Kk} \mathrm{k}_{12} \rho \mathrm{m}(\mathrm{s})+\mathrm{Kk} \mathrm{O}_{\mathrm{m}} \exp \left(\frac{\alpha}{\epsilon_{\mathrm{n}-\mathrm{m}}} \mathrm{s}\right)\right] \mathrm{ds} .
$$

From the above integral inequality, we obtain the following estimate.

$$
\mathrm{m}(\mathrm{t}) \leq \mathrm{K} \rho_{1} \exp \left[\frac{\mathrm{Kk}_{12} \rho}{\epsilon_{\mathrm{n}}-\mathrm{m}} \mathrm{t}\right]+\frac{\mathrm{Kk} \mathrm{O}_{\mathrm{m}}}{\alpha-\epsilon_{\mathrm{n}}-\mathrm{m} \mathrm{Kk}_{12}}\left[\exp \left[\frac{\alpha}{\epsilon_{\mathrm{n}}-\mathrm{m}} \mathrm{t}\right]-\exp \left[\frac{\mathrm{Kk}_{12} \rho}{\epsilon_{\mathrm{n}}-\mathrm{m}} \mathrm{t}\right]\right]
$$

From (5.11) and the above inequality, we will have,

Choose

$$
\left\|\Delta \mathrm{N}_{\mathrm{m}}\right\| \leq \mathrm{K} \rho_{1} \exp \left[-\frac{\alpha-\mathrm{Kk}_{12} \rho}{\epsilon_{\mathrm{n}}-\mathrm{m}} \mathrm{t}\right]+\mathrm{Kk} \frac{\mathrm{O}_{\mathrm{m}}}{\alpha-\mathrm{Kk}_{12} \rho}
$$

$$
\rho=\min \left(\frac{\alpha}{2 \mathrm{~K} \mathrm{k}_{12}}, \frac{4 \mathrm{Kk}}{\alpha} \mathrm{O}_{\mathrm{m}}\right)
$$

and

$$
\rho_{1}=\min \left(\frac{\rho}{4 \mathrm{~K}}, \frac{\rho}{4}\right)
$$

From the choice of $\rho_{1},(5.12)$, we get,

$$
\left\|\Delta \mathrm{N}_{\mathrm{m}}(\mathrm{t})\right\| \leq \rho=\sum_{\mathrm{k}=\mathrm{n}-\mathrm{m}}^{\mathrm{n}} \mathrm{O}\left(\frac{\epsilon_{\mathrm{k}}}{\epsilon_{\mathrm{k}-1}}\right)
$$

This completes the boundedness of solution of (5.7). It is obvious that $\mathrm{N}_{\mathrm{m}}$ defined by

$$
\mathrm{N}_{\mathrm{m}}(\mathrm{t})=\overline{\mathrm{N}}_{\mathrm{m}}(\mathrm{t})+\Delta \mathrm{N}_{\mathrm{m}}(\mathrm{t})
$$

is a solution of (5.2). This together with the boundedness of $\bar{N}_{m}$ and $\Delta N_{m}$ implies the existence of bounded solution $\mathrm{N}_{\mathrm{m}}$ of (5.2). Unstability of $\mathrm{F}_{11}(\mathrm{~m})+\mathrm{F}_{12}(\mathrm{~m}) \mathrm{N}_{\mathrm{m}}$ and stability of $\mathrm{F}_{22}(\mathrm{~m})-\mathrm{N}_{\mathrm{m}} \mathrm{F}_{12}(\mathrm{~m})$ follows directly from assumption $A 5.1$ and the approximation of $\mathrm{F}_{\mathrm{ij}}(\mathrm{m})$ and $\mathrm{N}_{\mathrm{m}}$ with $\hat{\mathrm{F}}_{\mathrm{ij}}(\mathrm{m})$ and $\overline{\mathrm{N}}_{\mathrm{m}}$.

Assumption A5.2: Algebraic equation corresponding to (5.3)

$$
0=\left(\hat{\mathrm{F}}_{11}(\mathrm{~m})+\hat{\mathrm{F}}_{12}(\mathrm{~m}) \overline{\mathrm{N}}_{\mathrm{m}}\right) \overline{\mathrm{P}}_{\mathrm{m}}-\overline{\mathrm{P}}_{\mathrm{m}}\left(\hat{\mathrm{F}}_{22}(\mathrm{~m})-\overline{\mathrm{N}}_{\mathrm{m}} \hat{\mathrm{F}}_{12}(\mathrm{~m})\right)+\hat{\mathrm{F}}_{12}(\mathrm{~m})
$$

has a bounded soslution $\overline{\mathrm{P}}_{\mathrm{m}}$, and $\overline{\mathrm{P}}_{\mathrm{m}}$ has a bounded derivative on $0 \leq t \leq 1$.

Theorem 5.2: Under the assumptions of Theorem 5.1 and A5.2, equation (5.3) has a solution $\mathrm{P}_{\mathrm{m}}$ and it can be approximated by solution $\overline{\mathrm{P}}_{\mathrm{m}}$ of (5.13).

Proof: Proof is analogous to the proof of Theorem 5.1. Therefore, we omit the details.

Now we will apply a transformation to the totally decoupled system (4.10) so that it decomposes every faster sub-systems into stable and unstable subsystems and leaves the slowest subsystem as it is. 
Let

$$
\mathrm{E}=\operatorname{diag}\left\{\mathrm{I}_{\mathrm{n}_{1}}, \mathrm{E}_{2}, \ldots, \mathrm{E}_{\mathrm{n}}\right\}
$$

We apply the transformation $\mathrm{E}$ defined by

$$
\left(\mathrm{w}_{1}^{\mathrm{T}} \mathrm{w}_{21}^{\mathrm{T}} \mathrm{w}_{22}^{\mathrm{T}} \ldots \mathrm{w}_{\mathrm{n} 1}^{\mathrm{T}} \mathrm{w}_{\mathrm{n} 2}^{\mathrm{T}}\right)^{\mathrm{T}}=\mathrm{E}\left(\mathrm{U}_{1}^{\mathrm{T}} \mathrm{U}_{21}^{\mathrm{T}} \mathrm{U}_{22}^{\mathrm{T}} \ldots \mathrm{U}_{\mathrm{n} 1}^{\mathrm{T}} \mathrm{U}_{\mathrm{n} 2}^{\mathrm{T}}\right)^{\mathrm{T}}
$$

to totally decoupled system (4.10) with boundary condition (4.11). Then, from (5.1)-(5.4), we will have

$$
\begin{gathered}
\dot{\mathrm{W}}_{1}=\mathrm{A}_{11}(\mathrm{n}-1) \mathrm{W}_{1} \\
\epsilon_{\mathrm{n}-\mathrm{m}}\left[\begin{array}{c}
\dot{\mathrm{W}}_{\mathrm{n}-\mathrm{m} 1} \\
\dot{\mathrm{W}}_{\mathrm{n}-\mathrm{m} 2}
\end{array}\right]=\left[\begin{array}{cc}
\mathrm{F}_{11}(\mathrm{~m})+\mathrm{F}_{12}(\mathrm{~m}) \mathrm{N}_{\mathrm{m}} & 0 \\
0 & \mathrm{~F}_{22}(\mathrm{~m})-\mathrm{N}_{\mathrm{m}} \mathrm{F}_{12}(\mathrm{~m})
\end{array}\right]\left[\begin{array}{l}
\mathrm{W}_{\mathrm{n}-\mathrm{m} \mathrm{1}} \\
\mathrm{W}_{\mathrm{n}-\mathrm{m} 2}
\end{array}\right], \mathrm{m} \in \mathrm{J}(0, \mathrm{n}-2) \\
\mathrm{Q}(\mathrm{n}-1, \epsilon) \mathrm{E}^{-1}(0) \mathrm{W}(0)+\mathrm{R}(\mathrm{n}-1, \epsilon) \mathrm{E}^{-1}(1) \mathrm{W}(1)=\mathrm{B}
\end{gathered}
$$

Now we establish the existence and uniqueness of a bounded solution of the totally decoupled system (4.10) with boundary condition (4.11) and the original boundary value problem (2.5) - (2.6). For this purpose we prove the exixtence and uniqueness of a bounded solution for the decomposed system (5.14) - (5.15).

Theorem 5.3: Let the assumptions A2.1, A4.1, A4.2, A4.3, A5.1 and A5.2 be satisfied. let $\nabla(\epsilon)$ defined by

$$
\begin{aligned}
\nabla(\epsilon)= & \mathrm{Q}(\mathrm{n}-1, \epsilon) \mathrm{E}^{-1}(0) \operatorname{diag}\left\{\phi_{1}(0,0), \phi_{21}(0,0), \phi_{22}(0,1), \ldots, \phi_{\mathrm{n} 1}(0,0), \phi_{\mathrm{n} 2}(0,1)\right\} \\
& +\mathrm{R}(\mathrm{n}-1, \epsilon) \mathrm{E}^{-1}(1) \operatorname{diag}\left\{\phi_{1}(1,0), \phi_{21}(1,0), \phi_{22}(1,0), \ldots, \phi_{\mathrm{n} 1}(1,0) \phi_{\mathrm{n} 2}(1,0)\right\}
\end{aligned}
$$

has an inverse, and its inverse is bounded.

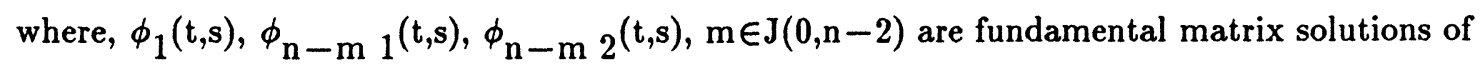

$$
\begin{aligned}
\dot{\mathrm{W}}_{1} & =\mathrm{A}_{11}(\mathrm{n}-1) \mathrm{W}_{1}, \\
\epsilon_{\mathrm{n}-\mathrm{m}} \dot{\mathrm{W}}_{\mathrm{n}-\mathrm{m} 1} & =\left[\mathrm{F}_{11}(\mathrm{~m})+\mathrm{F}_{12}(\mathrm{~m}) \mathrm{N}_{\mathrm{m}}\right] \mathrm{W}_{\mathrm{n}-\mathrm{m} 1}, \\
\epsilon_{\mathrm{n}-\mathrm{m}} \dot{\mathrm{W}}_{\mathrm{n}-\mathrm{m} 2} & =\left[\mathrm{F}_{22}(\mathrm{~m})-\mathrm{N}_{\mathrm{m}} \mathrm{F}_{12}(\mathrm{~m})\right] \mathrm{W}_{\mathrm{n}-\mathrm{m} 2}, \mathrm{~m} \in \mathrm{J}(0, \mathrm{n}-2),
\end{aligned}
$$




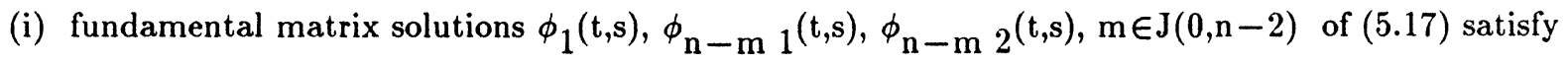

$$
\left\{\begin{array}{l}
\left\|\phi_{n-m 1}(t, s)\right\| \leq K_{n-m 1} \exp \left[-\frac{\alpha_{n-m} 1}{\epsilon_{n}-m}(s-t)\right], \text { for } 0 \leq t \leq s \leq 1, \\
\left\|\phi_{n-m 1}(t, s)\right\| \leq K_{n-m 2} \exp \left[-\frac{\alpha_{n-m}}{\epsilon_{n}-m}(t-s)\right], \text { for } 0 \leq s \leq t \leq 1, m \in J(0, n-2) .
\end{array}\right.
$$

and

$$
\left\|\phi_{1}(\mathrm{t}, \mathrm{s})\right\| \leq \mathrm{K}_{1} \exp \left[\alpha_{1}|\mathrm{t}-\mathrm{s}|\right] \text { for } 0 \leq \mathrm{t}, \mathrm{s} \leq 1
$$

where, for $\mathrm{m} \in \mathrm{J}(0, \mathrm{n}-2), \mathrm{K}_{\mathrm{n}-\mathrm{m} 1}, \mathrm{~K}_{\mathrm{n}-\mathrm{m} 2}, \alpha_{\mathrm{n}-\mathrm{m} 1}, \alpha_{\mathrm{n}-\mathrm{m} 2}, \mathrm{~K}_{1}$ and $\alpha_{1}$ are some positive integers, independent of $\epsilon_{\mathrm{n}-\mathrm{m}}$. Moreover

$$
\begin{gathered}
\mathrm{w}_{1}(\mathrm{t})=\phi_{1}(\mathrm{t}, 0) \eta_{1}, \\
\mathrm{~W}_{\mathrm{i} 1}(\mathrm{t})=\phi_{\mathrm{i} 1}(\mathrm{t}, 0) \eta_{\mathrm{i} 1}, \\
\mathrm{~W}_{\mathrm{i} 2}(\mathrm{t})=\phi_{\mathrm{i} 2}(\mathrm{t}, 1) \eta_{\mathrm{i} 2}, \mathrm{i} \in \mathrm{J}(2, \mathrm{n}),
\end{gathered}
$$

is a bounded solution of (5.14) for any $\left(\eta_{1}^{\mathrm{T}} \eta_{2}^{\mathrm{T}} \eta_{2}^{\mathrm{T}} \ldots \eta_{\mathrm{n} 1}^{\mathrm{T}} \eta_{\mathrm{n} 2}^{\mathrm{T}}\right)^{\mathrm{T}} \in \mathbb{R}^{\mathrm{N}}$;

(ii) the decomposed boundary value problem (5.14)-(5.15) has a unique bounded solution which can be expressed as (5.19) with

$$
\left(\eta_{1}^{\mathrm{T}} \eta_{2}^{\mathrm{T}} \eta_{2}^{\mathrm{T}}{ }_{2} \ldots \eta_{\mathrm{n} 1}^{\mathrm{T}} \eta_{\mathrm{n} 2}^{\mathrm{T}}\right)^{\mathrm{T}}=\nabla(\epsilon)^{-1} \mathrm{~B}(\epsilon)
$$

where $\nabla(\epsilon)$ is as defined in (5.16).

(iii) the original boundary value problem (2.5)-(2.6) has a unique bounded solution and it is given by

$$
X(t)=S^{2}(t) E^{-1}(t) W(t)
$$

where $\mathrm{S}^{2}$ as in Remark 4.2 and $\mathrm{W}(\mathrm{t})=\left(\mathrm{W}_{1}(\mathrm{t}) \mathrm{W}_{21}(\mathrm{t}) \mathrm{W}_{22}(\mathrm{t}) \ldots \mathrm{W}_{\mathrm{n} 1}(\mathrm{t}) \mathrm{W}_{\mathrm{n} 2}(\mathrm{t})\right)^{\mathrm{T}}$.

Proof: From the boundedness of $\mathrm{A}_{11}(\mathrm{n}-1)$ and from the stable, unstable modes as in Theorem 5.1, proof of (i) follows immediately. Let us prove (ii). From the choice of initial values, it can be shown that (5.19) satisfies the boundary condition (5.15). From (5.18) and from the boundedness of $\nabla(\epsilon)^{-1}$, we will have boundedness of the solution (5.19) of boundary value problem (5.14)-(5.15). Moreover, non-singularity of $\nabla(\epsilon)$ guarantees the unique choice of initial values. That is, it gurantees the uniqueness of solution of (5.14)-(5.15). This completes the proof of (ii). Now we complete the proof of the Theorem by proving (iii). Since $W(t)$ is the solution of the transformed decomposed system (5.14)-(5.15), $\mathrm{U}(\mathrm{t})=\mathrm{E}^{-1}(\mathrm{t}) \mathrm{W}(\mathrm{t})$ is the solution of the totally decoupled system (4.10) with (4.11). Since $\mathrm{S}^{2}=\left(\mathrm{T}^{2}\right)^{-1}$ transforms (4.10) with (4.11) to the original boundary value problem (2.5)-(2.6), $\mathrm{X}(\mathrm{t})=\mathrm{S}^{2}(\mathrm{t}) \mathrm{E}^{-1}(\mathrm{t}) \mathrm{W}(\mathrm{t})$ is the solution of (2.5)-(2.6). Boundedness of solution $\mathrm{X}(\mathrm{t})$ follows from 
the boundedness of $W(t), E^{-1}(t)$ and $S^{2}(t)$ for $0 \leq t \leq 1$. Uniqueness of the solution $X(t)$ follows from uniqueness of $U(t)$ and the non-singularity of $E$ and $T^{2}$.

Our objective in the rest of this section is to find an approximate solution of the totally decomposed system (5.14)-(5.15) by a solution of a boundary value problem with the given coefficient matrices and hence to find an approximate solution of the original boundary value problem (2.5)-(2.6). For this purpose let us consider an suxiliary system corresponding to the totally decomposed system (5.14) as follows;

$$
\begin{aligned}
& \dot{\hat{\mathrm{W}}}_{1}=\hat{\mathrm{A}}_{11}(\mathrm{n}-1) \hat{\mathrm{W}}_{1} \text {, }
\end{aligned}
$$

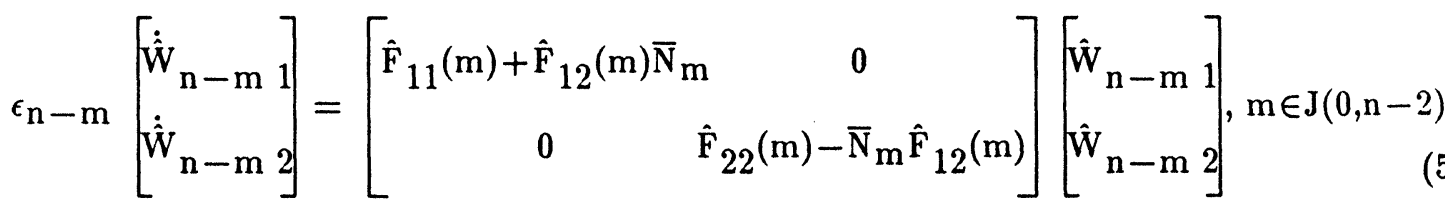

$$
\begin{aligned}
& \hat{\mathrm{Q}}(\mathrm{n}-1) \overline{\mathrm{E}}^{-1}(0) \hat{\mathrm{W}}(0)+\hat{\mathrm{R}}(\mathrm{n}-1) \overline{\mathrm{E}}^{-1}(1) \hat{\mathrm{W}}(1)=\mathrm{B}
\end{aligned}
$$

where $\overline{\mathrm{E}}=\operatorname{diag}\left\{\mathrm{I}_{\mathrm{n}_{1}}, \overline{\mathrm{E}}_{2}, \ldots, \overline{\mathrm{E}}_{\mathrm{n}}\right\}$

and

$$
\overline{\mathrm{E}}_{\mathrm{n}-\mathrm{m}}=\left[\begin{array}{cc}
\mathrm{I}_{\mathrm{k}_{\mathrm{m}}}+\overline{\mathrm{N}}_{\mathrm{m}} \overline{\mathrm{N}}_{\mathrm{m}} & -\overline{\mathrm{N}}_{\mathrm{m}} \\
-\overline{\mathrm{N}}_{\mathrm{m}} & \mathrm{I}_{\mathrm{n}_{\mathrm{n}-\mathrm{m}}-\mathrm{k}_{\mathrm{m}}}
\end{array}\right]
$$

We observe that the fundamental matrix solution of (5.20) approximates the fundamental matrix solution of (5.14). Now we assume the following assumptions.

Assumption A5.3: For $\mathrm{m} \in \mathrm{J}(0, \mathrm{n}-2)$, the coefficient matrices $\left(\hat{\mathrm{F}}_{11}(\mathrm{~m})+\hat{\mathrm{F}}_{12}(\mathrm{~m}) \overline{\mathrm{N}}_{\mathrm{m}}\right)$ and $\left(\hat{\mathrm{F}}_{22}(\mathrm{~m})-\overline{\mathrm{N}}_{\mathrm{m}} \hat{\mathrm{F}}_{12}(\mathrm{~m})\right)$ of $(5.20)$ satisfies lipchitz condition for $0 \leq \mathrm{t} \leq 1$.

Assumption A5.4: $\bar{\nabla}$ defined by,

$$
\bar{\nabla}=\hat{Q}(n-1) \overline{\mathrm{E}}^{-1}(0) \operatorname{diag}\left\{\mathrm{I}_{\mathrm{n}_{1}}, \mathrm{I}_{\mathrm{k}_{\mathrm{n}-2}}, 0, \ldots, \mathrm{I}_{\mathrm{k}_{0}}, 0\right\}+\hat{\mathrm{R}}(\mathrm{n}-1) \overline{\mathrm{E}}^{-1}(1) \operatorname{diag}\left\{\hat{\phi}_{1}(1,0), 0, \ldots, 0, \mathrm{I}_{\mathrm{n}_{\mathrm{n}}-\mathrm{k}_{0}}\right\}
$$

has bounded inverse, where $\hat{\phi}_{1}(t, s)$ is the fundamental matrix solution of

$$
\dot{\hat{\mathrm{W}}}_{1}=\mathrm{A}_{11}(\mathrm{n}-1) \hat{\mathrm{W}}_{1},
$$

Theorem 5.4: Let the assumptions A2.1, A4.1, A4.2, A4.3, A5.1, A5.2, A5.3 and A5.4 be satisfied. 
Then,

(i) the solution in (5.19) of (5.14)-(5.15) can be approximated by

$$
\begin{aligned}
\hat{\mathrm{W}}_{1}(\mathrm{t}) & =\hat{\phi}_{1}(\mathrm{t}, 0) \hat{\mathrm{W}}_{1}(0) \\
\hat{\mathrm{W}}_{\left.\mathrm{n}-\mathrm{m} 1^{(}\right)} & =\exp \left[\left(\hat{\mathrm{F}}_{11}(\mathrm{~m})+\hat{\mathrm{F}}_{12}(\mathrm{~m}) \overline{\mathrm{N}}_{\mathrm{m}}\right)(0) \frac{\mathrm{t}}{\epsilon_{\mathrm{n}-\mathrm{m}}}\right] \hat{\mathrm{W}}_{\mathrm{n}-\mathrm{m} \mathrm{1}}(0), \\
\hat{\mathrm{W}}_{\mathrm{n}-\mathrm{m} \mathrm{2}}(\mathrm{t}) & =\exp \left[\left(\hat{\mathrm{F}}_{22}(\mathrm{~m})-\overline{\mathrm{N}}_{\mathrm{m}} \hat{\mathrm{F}}_{12}(\mathrm{~m})\right)(1) \frac{(1-\mathrm{t})}{\epsilon_{\mathrm{n}-\mathrm{m}}}\right] \hat{\mathrm{W}}_{\mathrm{n}-\mathrm{m} \mathrm{1} 1}(1), \mathrm{m} \in \mathrm{J}(0, \mathrm{n}-2),
\end{aligned}
$$

where $\hat{\phi}_{1}(\mathrm{t}, 0)$ is as in assumption $\mathrm{A} 5.4$,

$$
\left(\hat{\mathrm{W}}_{1}^{0 \mathrm{~T}} \hat{\mathrm{W}}_{21}^{0 \mathrm{~T}} \hat{\mathrm{W}}_{22}^{1 \mathrm{~T}} \ldots \hat{\mathrm{W}}_{\mathrm{n} 1}^{0 \mathrm{~T}} \hat{\mathrm{W}}_{\mathrm{n} 2}^{1 \mathrm{~T}}\right)^{\mathrm{T}}=\bar{\nabla}^{-1} \mathrm{~B}
$$

and

$$
\left(\hat{\mathrm{F}}_{11}(\mathrm{~m})+\hat{\mathrm{F}}_{12}(\mathrm{~m}) \mathrm{N}_{\mathrm{m}}\right)(\mathrm{i})=\hat{\mathrm{F}}_{11}(\mathrm{~m}, \mathrm{i})+\hat{\mathrm{F}}_{12}(\mathrm{~m}, \mathrm{i}) \mathrm{N}_{\mathrm{m}}(\mathrm{i}), \text { for } \mathrm{i}=0,1
$$

(ii) the solution $\mathrm{X}(\mathrm{t})$ of the original boundary value problem (2.5)-(2.6) can be approximated by $\hat{S}^{2}(t) \bar{E}^{-1}(t) \hat{W}(t)$.

Proof: From Theorems 5.1 and 5.2, we observe that $\mathrm{E}(\mathrm{t})$ can be approximated by $\overline{\mathrm{E}}(\mathrm{t})$. Furthermore, we notice that the fundamental matrix solutions of (5.14) can be approximated by the fundamental matrix solutions given in (5.22). To complete the proof of (i) it is enough to show $\eta_{\mathrm{ij}}$ 's $\mathrm{i} \in \mathrm{J}(2, \mathrm{n})$, $\mathrm{j} \in \mathrm{J}(1,2)$ in (5.19) can be approximated by $\hat{\mathrm{W}}_{\mathrm{i}}{ }_{1}^{0} \hat{\mathrm{W}}_{\mathrm{i}} \frac{1}{2}, \mathrm{i} \in \mathrm{J}(2, \mathrm{n})$, resspectively. This statement follows from the fact that

$$
\phi_{\mathrm{i} 2}(0,1) \cong \sum_{k=n-i}^{n} O\left(\frac{{ }^{\epsilon} k}{\epsilon-1}\right) \text {, for } i \in J(0, n-2) .
$$

Let us prove (ii). From Theorem 5.3, the solution of he original boundary value problem (2.5)-(2.6) is $\mathrm{X}(\mathrm{t})=\mathrm{S}^{2}(\mathrm{t}) \mathrm{E}(\mathrm{t}) \mathrm{W}(\mathrm{t})$. From Remark 4.4, $\hat{\mathrm{S}}^{2}(\mathrm{t})$ approximates $\mathrm{S}^{2}(\mathrm{t})$, from (i) $\overline{\mathrm{E}}(\mathrm{t}), \overline{\mathrm{W}}(\mathrm{t})$ approximates $E(t), W(t)$ respectively. Hence $\hat{S}^{2}(t) \bar{E}^{-1}(t) \hat{W}(t)$ approximates $X(t)$. This compleres the proof of the theorem.

Remark 5.1: We note that the dichotomy transformation is determined by the system of differential equation in (5.2) and (5.3). The results of this section generalize the results of Wilde and Kokotovic $[17,18]$ in a systematic way. This fact can be justified in the succeeding sections. 


\section{Application}

In this section we consider an optimal control problem. The preceeding results are applied to find an approximate solution of the boundary value problem associated with the necessary optimality condition.

Let us consider an optimal control problem.

$$
\begin{aligned}
\dot{\mathrm{X}}_{1} & =\mathrm{A}_{11}(\mathrm{t}) \mathrm{X}_{1}+\mathrm{A}_{12}(\mathrm{t}) \mathrm{X}_{2}+\mathrm{B}_{1}(\mathrm{t}) \mathrm{U} \\
\epsilon_{2} \dot{\mathrm{X}}_{2} & =\mathrm{A}_{21}(\mathrm{t}) \mathrm{X}_{1}+\mathrm{A}_{22}(\mathrm{t}) \mathrm{X}_{2}+\mathrm{B}_{2}(\mathrm{t}) \mathrm{U} .
\end{aligned}
$$

with the performance index

$$
J=\frac{1}{2}\left[\left.\mathrm{X}_{1}^{\mathrm{T}} \mathrm{X}_{2}^{\mathrm{T}}\left[\begin{array}{cc}
\mathrm{I}_{\mathrm{n}_{1}} & 0 \\
0 & \epsilon_{2} \mathrm{I}_{2}
\end{array}\right] \Pi^{\epsilon}\left[\begin{array}{c}
\mathrm{X}_{1} \\
\mathrm{X}_{2}
\end{array}\right]\right|_{\mathrm{t}=1}+\frac{1}{2} \int_{0}^{1}\left\{\left[\mathrm{X}_{1}^{\mathrm{T}} \mathrm{X}_{2}^{\mathrm{T}}\right] \mathrm{Q}(\mathrm{t})\left[\begin{array}{c}
\mathrm{X}_{1} \\
\mathrm{X}_{2}
\end{array}\right]+\mathrm{U}^{\mathrm{T}} \mathrm{R}(\mathrm{t}) \mathrm{U}\right\} \mathrm{dt}\right.
$$

and

$$
\left[\mathrm{X}_{1}^{\mathrm{T}}(0) \mathrm{X}_{2}^{\mathrm{T}}(0)\right]=\left[\mathrm{X}_{1}^{0 \mathrm{~T}} \mathrm{X}_{2}^{0} \mathrm{~T}\right]
$$

where state variables $\mathrm{X}_{1}, \mathrm{X}_{2}$ and control variables $\mathrm{U}$ are of $\mathrm{n}_{1}, \mathrm{n}_{2}$ and $\mathrm{m}$ dimensional vectors, respectively; $\Pi^{\epsilon} 2$ is positive semidefinite matrix for all $0 \leq \epsilon_{2} \leq \epsilon_{2}^{*}$, and

$$
\Pi^{\epsilon}=\left[\begin{array}{cc}
\Pi_{11} & \epsilon_{2} \Pi_{12} \\
\Pi_{12}^{\mathrm{T}} & \Pi_{22}
\end{array}\right], \mathrm{Q}=\left[\begin{array}{ll}
\mathrm{Q}_{11}(\mathrm{t}) & \mathrm{Q}_{12}(\mathrm{t}) \\
\mathrm{Q}_{12}(\mathrm{t}) & \mathrm{Q}_{22}(\mathrm{t})
\end{array}\right], \mathrm{R}=\left[\begin{array}{cc}
\mathrm{R}_{11}(\mathrm{t}) & \mathrm{R}_{12}(\mathrm{t}) \\
\mathrm{R}_{12}(\mathrm{t}) & \mathrm{R}_{22}(\mathrm{t})
\end{array}\right]
$$

Assumption A 6.1: For all $0 \leq t \leq 1,0 \leq \epsilon_{2} \leq \epsilon_{2}^{*}$, the coefficient matrices $A_{i j}, Q_{i j}, R_{i j}$ for $i, j \in J(1,2) ; B_{i}$ for $i \in J(1,2)$ are twice continuously differentiable with respect to $t$. Furthermore, we assume that $R$ is symmetric positive definite and $Q$ is symmetric positive semi definite.

With the assumption $A$ 6.1 , for the necessary optimality condition, the variables $\left[\mathrm{X}_{1}^{\mathrm{T}} \mathrm{X}_{2}^{\mathrm{T}}\right]^{\mathrm{T}}$ and the adjoint variables $\left[\lambda_{1}^{\mathrm{T}} \lambda_{2}^{\mathrm{T}}\right]^{\mathrm{T}}$ must satisfy the follwing boundary value problem $[1,17]$ :

$$
\begin{gathered}
{\left[\begin{array}{c}
\dot{\mathrm{x}}_{1} \\
\dot{\lambda}_{1}
\end{array}\right]=\left[\begin{array}{cc}
\mathrm{A}_{11} & -\mathrm{S}_{11} \\
-\mathrm{Q}_{11} & -\mathrm{A}_{11}^{\mathrm{T}}
\end{array}\right]\left[\begin{array}{c}
\mathrm{x}_{1} \\
\lambda_{1}
\end{array}\right]+\left[\begin{array}{cc}
\mathrm{A}_{12} & -\mathrm{S}_{12} \\
-\mathrm{Q}_{12} & -\mathrm{A}_{21}^{\mathrm{T}}
\end{array}\right]\left[\begin{array}{c}
\mathrm{x}_{2} \\
\lambda_{2}
\end{array}\right]} \\
\epsilon_{2}\left[\begin{array}{c}
\dot{\mathrm{x}}_{2} \\
\dot{\lambda}_{2}
\end{array}\right]=\left[\begin{array}{ll}
\mathrm{A}_{21} & -\mathrm{S}_{21} \\
-\mathrm{Q}_{21} & -\mathrm{A}_{21}^{\mathrm{T}}
\end{array}\right]\left[\begin{array}{c}
\mathrm{x}_{1} \\
\lambda_{1}
\end{array}\right]+\left[\begin{array}{ll}
\mathrm{A}_{22} & -\mathrm{S}_{22} \\
-\mathrm{Q}_{22} & -\mathrm{A}_{22}^{\mathrm{T}}
\end{array}\right]\left[\begin{array}{c}
\mathrm{x}_{2} \\
\lambda_{2}
\end{array}\right]
\end{gathered}
$$


with boundary condition

$$
\mathrm{H}_{1} \mathrm{X}(0)+\mathrm{H}_{2} \mathrm{X}(1)=\mathrm{H}_{3}
$$

where

$$
\begin{aligned}
& \mathrm{H}_{1}=\left[\begin{array}{cccc}
\mathrm{I}_{\mathrm{n}_{1}} & 0 & 0 & 0 \\
0 & 0 & 0 & 0 \\
0 & 0 & \mathrm{I}_{2} & 0 \\
0 & 0 & 0 & 0
\end{array}\right], \quad \mathrm{H}_{2}=\left[\begin{array}{cccc}
0 & 0 & 0 & 0 \\
-\Pi_{11} & \mathrm{I}_{\mathrm{n}_{1}} & -\epsilon_{2} \Pi_{12} & 0 \\
0 & 0 & 0 & 0 \\
-\Pi_{12}^{\mathrm{T}} & 0 & -\Pi_{22} & \mathrm{I}_{\mathrm{n}_{2}}
\end{array}\right], \mathrm{H}_{3}=\left[\begin{array}{c}
\mathrm{x}_{1}^{0} \\
0 \\
\mathrm{x}_{2}^{0} \\
0
\end{array}\right] \\
& \mathrm{X}(0)=\left[\begin{array}{c}
\mathrm{x}_{1}^{0} \\
\lambda_{1}^{0} \\
\mathrm{X}_{2}^{0} \\
\lambda_{2}^{0}
\end{array}\right], \mathrm{X}(1)=\left[\begin{array}{c}
\mathrm{x}_{1}^{1} \\
\lambda_{1}^{1} \\
\mathrm{x}_{2}^{1} \\
\lambda_{2}^{1}
\end{array}\right] \text { and }\left[\begin{array}{cc}
\mathrm{S}_{11} & \mathrm{~S}_{12} \\
\mathrm{~S}_{21} & \mathrm{~S}_{22}
\end{array}\right]=\left[\begin{array}{c}
\mathrm{B}_{1} \\
\mathrm{~B}_{2}
\end{array}\right] \mathrm{R}^{-1}\left[\begin{array}{cc}
\mathrm{B}_{1}^{\mathrm{T}} & \mathrm{B}_{2}^{\mathrm{T}}
\end{array}\right]
\end{aligned}
$$

Assumption A 6.2: For all $t \in[0,1]$,

$$
\begin{aligned}
& \operatorname{rank}\left[\mathrm{B}_{2}, \mathrm{~A}_{22} \mathrm{~B}_{2}, \ldots, \mathrm{A}_{22}^{\mathrm{n}-2} \mathrm{~B}_{2}\right]=\mathrm{n}_{2}, \\
& \operatorname{rank}\left[\mathrm{C}_{2}^{\mathrm{T}}, \mathrm{A}_{22}^{\mathrm{T}} \mathrm{C}_{2}, \ldots, \mathrm{A}_{22}^{\mathrm{T}} \mathrm{n}-2 \mathrm{C}_{2}\right]=\mathrm{n}_{2},
\end{aligned}
$$

where $\mathrm{C}_{2}$ satisfies $\mathrm{C}_{2}^{\mathrm{T}} \mathrm{C}_{2}=\mathrm{Q}_{22}$.

Remark 6.1: From the assumptions A 6.1 and A 6.2, it is known [18,19] that the algebraic system

$$
0=-\mathrm{KA}_{22}-\mathrm{A}_{22}^{\mathrm{T}} \mathrm{K}+\mathrm{KS}_{22} \mathrm{~K}-\mathrm{Q}_{22}
$$

has a symmetric negative definite solution $\bar{N}$, and $-\left(A_{22}-S_{22} \bar{N}\right)$ is stable. From this, it can be observed that

$\left[\begin{array}{cc}A_{22} & -S_{22} \\ -Q_{22} & -A_{22}^{T}\end{array}\right]^{-1}=\left[\begin{array}{cc}\mathrm{D}^{-1}+\mathrm{D}^{-1} \mathrm{~S}_{22} \mathrm{D}^{-1 \mathrm{~T}} \overline{\mathrm{N}} & -\mathrm{D}^{-1} \mathrm{~S}_{22} \mathrm{D}^{-1 \mathrm{~T}} \\ \overline{\mathrm{N}} \mathrm{D}^{-1}+\overline{\mathrm{N}} \mathrm{D}^{-1} \mathrm{~S}_{22} \mathrm{D}^{-1 \mathrm{~T}} \overline{\mathrm{N}}+\mathrm{D}^{-1 \mathrm{~T}} \overline{\mathrm{N}} & -\mathrm{D}^{-1 \mathrm{~T}}-\overline{\mathrm{N}} \mathrm{D}^{-1} \mathrm{~S}_{22} \mathrm{D}^{-1 \mathrm{~T}}\end{array}\right]$

where $\mathrm{D}=\mathrm{A}_{22}-\mathrm{S}_{22} \overline{\mathrm{N}}$.

From assumptions A6.1, A6.2 and Remark 6.1, it is obvious that assumptions A4.1, A4.2 and 
A4.3 are valid relative to system $(6.3)-(6.4)$. Therefore, the decoupled system corresponding to this system $(6.3)-(6.4)$ is given by

$$
\begin{aligned}
& {\left[\begin{array}{c}
\dot{\mathrm{U}}_{1} \\
\dot{\mathrm{U}}_{2}
\end{array}\right]=\left\{\left[\begin{array}{cc}
\mathrm{A}_{11} & -\mathrm{S}_{11} \\
-\mathrm{Q}_{11} & -\mathrm{A}_{11}^{\mathrm{T}}
\end{array}\right]+\left[\begin{array}{cc}
\mathrm{A}_{12} & -\mathrm{S}_{12} \\
-\mathrm{Q}_{12} & -\mathrm{A}_{21}^{\mathrm{T}}
\end{array}\right]\left[\begin{array}{cc}
\mathrm{L}_{11} & \mathrm{~L}_{12} \\
\mathrm{~L}_{21} & \mathrm{~L}_{22}
\end{array}\right]\right\}\left[\begin{array}{c}
\mathrm{U}_{1} \\
\mathrm{U}_{2}
\end{array}\right]} \\
& \epsilon_{2}\left[\begin{array}{c}
\dot{\mathrm{U}}_{3} \\
\dot{\mathrm{U}}_{4}
\end{array}\right]=\left\{\left[\begin{array}{ll}
\mathrm{A}_{22} & -\mathrm{S}_{22} \\
-\mathrm{Q}_{22} & -\mathrm{A}_{22}^{\mathrm{T}}
\end{array}\right]-\epsilon_{2}\left[\begin{array}{ll}
\mathrm{L}_{11} & \mathrm{~L}_{12} \\
\mathrm{~L}_{21} & \mathrm{~L}_{22}
\end{array}\right]\left[\begin{array}{cc}
\mathrm{A}_{12} & -\mathrm{S}_{12} \\
-\mathrm{Q}_{12} & -\mathrm{A}_{21}^{\mathrm{T}}
\end{array}\right]\right\}\left[\begin{array}{c}
\mathrm{U}_{3} \\
\mathrm{U}_{4}
\end{array}\right]
\end{aligned}
$$

with the boundary condition

$$
\mathrm{H}_{1} \mathrm{~T}^{-1}(0) \mathrm{U}(0)+\mathrm{H}_{2} \mathrm{~T}^{-1}(1) \mathrm{U}(1)=\mathrm{H}_{3}
$$

where

and

$$
\mathrm{T}=\left[\begin{array}{cc}
\mathrm{I}_{2 \mathrm{n}_{1}}+\epsilon_{2} \mathrm{ML} & -\epsilon_{2} \mathrm{M} \\
-\mathrm{L} & \mathrm{I}_{2 \mathrm{n}_{2}}
\end{array}\right], \mathrm{L}=\left[\begin{array}{cc}
\hat{\mathrm{L}}_{11} & \hat{\mathrm{L}}_{12} \\
\hat{\mathrm{L}}_{21} & \hat{\mathrm{L}}_{22}
\end{array}\right]+\mathrm{O}\left(\epsilon_{2}\right)
$$

$$
\left[\begin{array}{ll}
\hat{\mathrm{L}}_{11} & \hat{\mathrm{L}}_{12} \\
\hat{\mathrm{L}}_{21} & \hat{\mathrm{L}}_{22}
\end{array}\right]=\left[\begin{array}{cc}
\mathrm{A}_{22} & -\mathrm{S}_{22} \\
-\mathrm{Q}_{22} & -\mathrm{A}_{22}^{\mathrm{T}}
\end{array}\right]^{-1}\left[\begin{array}{cc}
\mathrm{A}_{21} & -\mathrm{S}_{21} \\
-\mathrm{Q}_{21} & -\mathrm{A}_{21}^{\mathrm{T}}
\end{array}\right]
$$

Furthermore, we remark that the assumptions A6.1 and A6.2, in the context of Remark 6.1, imply the validity of assumptions A5.1 and A5.2. This together with the applications of Theorems 5.1 and 5.2, a totally decomposed system similar to $(5.20)$ can be written as

$$
\begin{aligned}
& {\left[\begin{array}{c}
\dot{\hat{\mathrm{W}}}_{1} \\
\dot{\hat{\mathrm{W}}}_{2}
\end{array}\right]=\left[\begin{array}{cc}
\mathrm{G}_{11} & \mathrm{G}_{12} \\
\mathrm{G}_{21} & \mathrm{G}_{22}
\end{array}\right]\left[\begin{array}{c}
\hat{\mathrm{W}}_{1} \\
\hat{\mathrm{W}}_{2}
\end{array}\right]} \\
& \epsilon_{2}\left[\begin{array}{c}
\dot{\hat{\mathrm{W}}}_{3} \\
\dot{\hat{\mathrm{W}}}_{4}
\end{array}\right]=\left[\begin{array}{cc}
\mathrm{A}_{22}-\mathrm{S}_{22} \mathrm{~N} & 0 \\
0 & -\left(\mathrm{A}_{22}^{\mathrm{T}}-\mathrm{N} \mathrm{S}_{22}\right)
\end{array}\right]\left[\begin{array}{c}
\hat{\mathrm{W}}_{3} \\
\hat{\mathrm{W}}_{4}
\end{array}\right]
\end{aligned}
$$




$$
\mathrm{H}_{1} \hat{\mathrm{T}}^{-1}(0) \overline{\mathrm{E}}(0) \hat{\mathrm{W}}(0)+\mathrm{H}_{2} \hat{\mathrm{T}}^{-1}(1) \overline{\mathrm{E}}(1) \hat{\mathrm{W}}(1)=\mathrm{H}_{3}
$$

where

$\mathrm{H}_{1} \hat{\mathrm{T}}^{-1}(0) \overline{\mathrm{E}}(0)=\left[\begin{array}{cccc}\mathrm{I}_{\mathrm{n}_{1}} & 0 & 0 & 0 \\ 0 & 0 & 0 & 0 \\ \mathrm{H}_{2} \hat{\mathrm{T}}^{-1}(1) \overline{\mathrm{E}}(1)= & \hat{\mathrm{L}}_{12}(0) & \mathrm{I}_{\mathrm{n}_{2}} & \mathrm{P}(0) \\ 0 & 0 & 0 & 0\end{array}\right]$,

$\left[\begin{array}{cccc}0 & 0 & 0 & 0 \\ -\Pi_{11} & \mathrm{I}_{\mathrm{n}_{1}} & 0 & 0 \\ 0 & 0 & 0 & 0 \\ \begin{array}{c}\Pi_{12}^{\mathrm{T}}-\Pi_{22} \hat{\mathrm{L}}_{11}(1) \\ +\hat{\mathrm{L}}_{21}(1)\end{array} & \begin{array}{c}-\Pi_{22} \hat{\mathrm{L}}_{12}(1) \\ +\hat{\mathrm{L}}_{22}(1)\end{array} & -\Pi_{22}+\mathrm{N}(1) & \begin{array}{c}-\Pi_{22} \mathrm{P}(1)+\mathrm{I}_{\mathrm{n}_{2}} \\ +\mathrm{P}(1) \mathrm{N}(1)\end{array}\end{array}\right]$

and

$$
\left[\begin{array}{ll}
G_{11} & G_{12} \\
G_{21} & G_{22}
\end{array}\right]=\left[\begin{array}{cc}
A_{11} & -S_{11} \\
-Q_{11} & -A_{11}^{T}
\end{array}\right]-\left[\begin{array}{cc}
A_{12} & -S_{12} \\
-Q_{12} & -A_{21}^{T}
\end{array}\right]
$$

In order to apply Theorem 5.4, we need to verify the assumptions A5.3 and A5.4. We note that assumption A6.1 implies A5.3. Therefore, we need to verify the validity of assumption A5.4. For this purpose, it is enough to verify the invertibility of the following matrix.

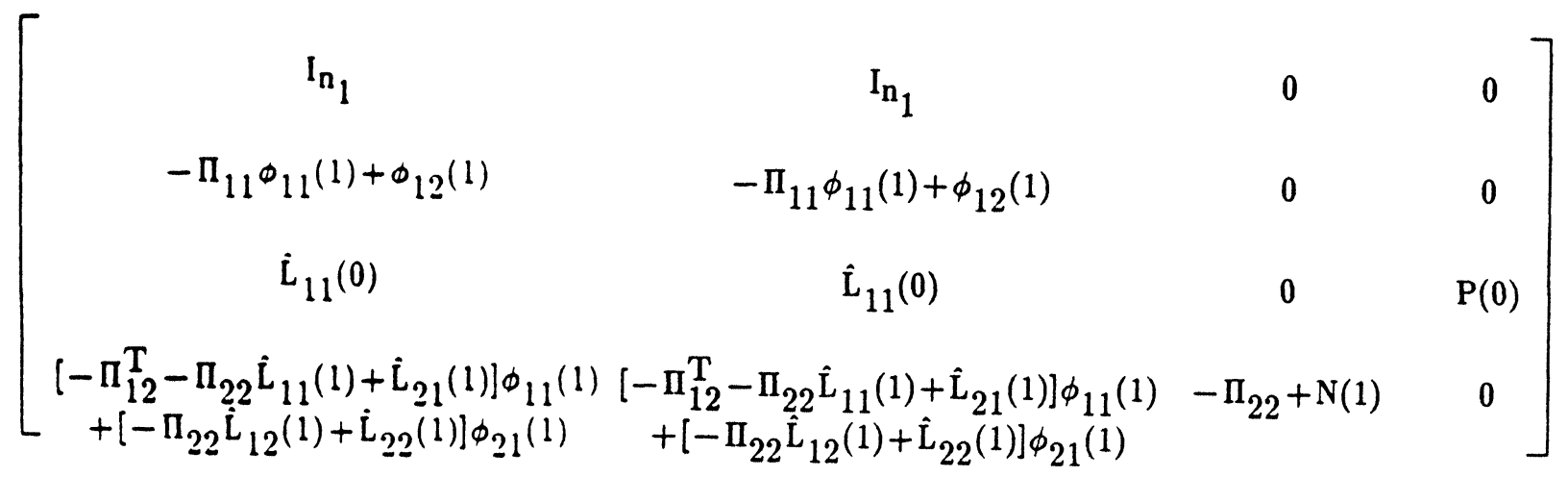


where $\quad\left[\begin{array}{ll}\phi_{11}(\mathrm{t}) & \phi_{12}(\mathrm{t}) \\ \phi_{21}(\mathrm{t}) & \phi_{22}(\mathrm{t})\end{array}\right]$ is the fundamental matrix solution of the slow system in (6.8).

Since $P(0)$ and $-\Pi_{22}+N(1)$ are invertible, it is enough to verify the invertibility of

$\left[\begin{array}{cc}\mathrm{I}_{\mathrm{n}_{1}} & 0 \\ -\Pi_{11_{11}(1)} & -\Pi_{11^{\prime} \phi_{12}(1)} \\ +\phi_{21}(1) & +\phi_{22}(1)\end{array}\right]=\left[\begin{array}{cc}\mathrm{I}_{\mathrm{n}_{1}} & 0 \\ 0 & 0\end{array}\right]+\left[\begin{array}{cc}0 & 0 \\ -\Pi_{11} & \mathrm{I}_{\mathrm{n}_{1}}\end{array}\right]\left[\begin{array}{cc}\phi_{11}(1) & \phi_{12}(1) \\ \phi_{21}(1) & \phi_{22}(1)\end{array}\right]$.

To verify the above statement, we present the following lemma.

Lemma 6.1: Let the assumptions A6.1 and A6.2 hold. Then the recatti differential equation

$$
\dot{\mathrm{K}}_{1}=-\mathrm{K}_{1} \mathrm{G}_{11}-\mathrm{G}_{11}^{\mathrm{T}} \mathrm{K}_{1}+\mathrm{K}_{1} \mathrm{G}_{12} \mathrm{~K}_{1}-\mathrm{G}_{21}, \mathrm{~K}_{1}=\Pi_{11}
$$

has a solution. Moreover, matrix given in equation (6.11) is invertible.

Proof: From the structure of $G_{i j}, i, j=1,2$ in $(6.10)$, after several algebraic computations, we can conclude that

$$
\left\{\begin{array}{l}
\mathrm{G}_{22}=-\mathrm{G}_{11}^{\mathrm{T}} \\
\mathrm{G}_{12}=-\left[\mathrm{B}_{11}+\mathrm{S}_{12} \mathrm{KD}^{-1} \mathrm{~B}_{12}-\mathrm{A}_{12} \mathrm{D}^{-1} \mathrm{~B}_{12}\right] \mathrm{R}^{-1}\left[\mathrm{~B}_{11}+\mathrm{S}_{12} \mathrm{KD}^{-1} \mathrm{~B}_{12}-\mathrm{A}_{12} \mathrm{D}^{-1} \mathrm{~B}_{12}\right]^{\mathrm{T}} \\
\mathrm{G}_{21}=-\left[\mathrm{Q}_{11}+\left(\xi_{1}+\xi_{2}\right) \mathrm{Q}_{12}^{\mathrm{T}}+\mathrm{Q}_{12}\left(\xi_{1}^{\mathrm{T}}+\xi_{2}^{\mathrm{T}}\right)+\left(\xi_{1}+\xi_{2}\right) \mathrm{Q}_{22}\left(\xi_{1}^{\mathrm{T}}+\xi_{2}^{\mathrm{T}}\right)\right]
\end{array}\right.
$$

where

$$
\xi_{1}=\mathrm{Q}_{12} \mathrm{D}^{-1} \mathrm{~K}^{-1}, \xi_{2}=\mathrm{A}_{12}^{\mathrm{T}} \mathrm{KD}^{-1} \mathrm{~K}^{-1} \text {. }
$$

From the above identities (6.13) and (6.14), it is known that $[18,19],(6.12)$ has a solution. To complete the proof of the lemma, let us consider the following differential equation

$$
\left[\begin{array}{c}
\dot{\mathrm{W}}_{1} \\
\dot{\hat{\mathrm{W}}}_{2}
\end{array}\right]=\left[\begin{array}{cc}
\mathrm{G}_{11} & \mathrm{G}_{12} \\
\mathrm{G}_{21} & \mathrm{G}_{22}
\end{array}\right]\left[\begin{array}{c}
\hat{\mathrm{W}}_{1} \\
\hat{\mathrm{W}}_{2}
\end{array}\right]
$$

Now we apply a transformation defined by

$$
\left[\begin{array}{c}
\mathrm{z}_{1} \\
\mathrm{z}_{2}
\end{array}\right]=\mathrm{T}_{1}\left[\begin{array}{c}
\hat{\mathrm{W}}_{1} \\
\hat{\mathrm{W}}_{2}
\end{array}\right]
$$


to totally decouple the system $(6.15)$,

where

$$
\mathrm{T}_{1}=\left[\begin{array}{cc}
\mathrm{I}_{\mathrm{n}_{1}}+\mathrm{K}_{1} \mathrm{~K}_{2} & -\mathrm{K}_{2} \\
-\mathrm{K}_{1} & \mathrm{I}_{\mathrm{n}_{1}}
\end{array}\right]
$$

This decoupling procedure requires that $K_{1}$ satisfy equation (6.12) and $K_{2}$ satisfy the following differential equation

$$
\dot{\mathrm{K}}_{2}=\left(\mathrm{G}_{11}+\mathrm{G}_{12} \mathrm{~K}_{1}\right) \mathrm{K}_{2}+\mathrm{K}_{2}\left(\mathrm{G}_{11}^{\mathrm{T}}+\mathrm{K}_{1} \mathrm{G}_{12}\right)+\mathrm{G}_{12}, \mathrm{~K}_{2}(0)=\mathrm{K}_{2}^{0} .
$$

Then we will have

$$
\left[\begin{array}{c}
\dot{\mathrm{Z}}_{1} \\
\dot{\mathrm{Z}}_{2}
\end{array}\right]=\left[\begin{array}{ccc}
\mathrm{G}_{11}-\mathrm{G}_{12} \mathrm{~K}_{1} & 0 \\
0 & -\left(\mathrm{G}_{11}^{\mathrm{T}}-\mathrm{K}_{1} \mathrm{G}_{12}\right)
\end{array}\right]\left[\begin{array}{c}
\mathrm{z}_{1} \\
\mathrm{z}_{2}
\end{array}\right]
$$

A fundamental matrix solution of the above linear system is given as

$$
\left[\begin{array}{cc}
\Psi_{11}(\mathrm{t}, 0) & 0 \\
0 & \Psi_{22}(\mathrm{t}, 0)
\end{array}\right]
$$

From this and (6.16), a fundamental matrix of $(6.15)$ is of the form

$$
\left[\begin{array}{cc}
\phi_{11}(\mathrm{t}) & \phi_{12}(\mathrm{t}) \\
\phi_{21}(\mathrm{t}) & \phi_{22}(\mathrm{t})
\end{array}\right]=\left[\begin{array}{cc}
\mathrm{I}_{\mathrm{n}_{1}}+\mathrm{K}_{1}(\mathrm{t}) \mathrm{K}_{2}(\mathrm{t}) & -\mathrm{K}_{2}(\mathrm{t}) \\
-\mathrm{K}_{1}(\mathrm{t}) & \mathrm{I}_{\mathrm{n}_{1}}
\end{array}\right]\left[\begin{array}{cc}
\Psi_{11}(\mathrm{t}, 0) & 0 \\
0 & \Psi_{22}(\mathrm{t}, 0)
\end{array}\right]\left[\begin{array}{cc}
\mathrm{I}_{\mathrm{n}_{1}} & \mathrm{~K}_{2}(0) \\
\mathrm{K}_{1}(0) & \mathrm{I}_{\mathrm{n}_{1}}+\mathrm{K}_{1}(0) \mathrm{K}_{2}(0)
\end{array}\right]
$$

This together with $\mathrm{K}_{1}(1)=\Pi_{11}$, yields

$$
\left[\begin{array}{cc}
\mathrm{I}_{\mathrm{n}_{1}} & 0 \\
-\Pi_{11} \phi_{11}(1) & -\Pi_{11} \phi_{12}(1) \\
+\phi_{21}(1) & +\phi_{22}(1)
\end{array}\right]=\left[\begin{array}{cc}
\mathrm{I}_{\mathrm{n}_{1}} & \mathrm{~K}_{2}(1) \Psi_{22}(1) \\
0 & \Psi_{2}(1)
\end{array}\right]\left[\begin{array}{cc}
\mathrm{I}_{\mathrm{n}_{1}} & \mathrm{~K}_{2}(0) \\
\mathrm{K}_{1}(0) & \mathrm{I}_{\mathrm{n}_{1}}+\mathrm{K}_{1}(0) \mathrm{K}_{2}(0)
\end{array}\right]
$$

It is obvious that the above matrix is invertible. This completes the proof of the lemma. Now by the application of Theorem 5.4, the expression in (5.23) and (5.24) with respect to (6.2) and (6.3) are given by 


$$
\begin{aligned}
& \hat{\mathrm{W}}_{1}(0)=\mathrm{X}_{1}^{0}, \\
& \hat{\mathrm{W}}_{2}(0)=\left[\Pi_{11} \phi_{12}(1)-\phi_{22}(1)\right]^{-1}\left[-\Pi_{11} \phi_{11}(1)+\phi_{21}(1)\right] \mathrm{X}_{1}^{0}, \\
& \hat{\mathrm{W}}_{3}(0)=\mathrm{P}^{-1}(0)\left[\mathrm{x}_{2}^{0}-\overline{\mathrm{X}}_{2}^{0}\right], \\
& \hat{\mathrm{W}}_{4}(0)=\left[\Pi_{22}-\mathrm{N}(1)\right]^{-1}\left\{\bar{\lambda}_{2}(1)-\Pi_{12}^{\mathrm{T}} \overline{\mathrm{X}}_{1}(1)-\Pi_{22} \overline{\mathrm{X}}_{2}(1)\right\}
\end{aligned}
$$

and for $0 \leq \mathrm{t} \leq 1$,

$$
\begin{aligned}
& \mathrm{X}_{1}(\mathrm{t})=\overline{\mathrm{X}}_{1}(\mathrm{t})+\mathrm{O}\left(\epsilon_{2}\right) \\
& \lambda_{1}(\mathrm{t})=\bar{\lambda}_{1}(\mathrm{t})+\mathrm{O}\left(\epsilon_{2}\right) \\
& \mathrm{X}_{2}(\mathrm{t})=\overline{\mathrm{X}}_{2}(\mathrm{t})+\hat{\mathrm{W}}_{3}(\mathrm{t})+\mathrm{P}(\mathrm{t}) \hat{\mathrm{W}}_{4}(\mathrm{t})+\mathrm{O}\left(\epsilon_{2}\right) \\
& \lambda_{2}(\mathrm{t})=\bar{\lambda}_{2}(\mathrm{t})+\mathrm{N}(\mathrm{t}) \hat{\mathrm{W}}_{3}(\mathrm{t})+\left[\mathrm{I}_{2}+\mathrm{N}(\mathrm{t}) \mathrm{P}(\mathrm{t})\right] \hat{\mathrm{W}}_{4}(\mathrm{t})+\mathrm{O}\left(\epsilon_{2}\right)
\end{aligned}
$$

where

$$
\begin{aligned}
\overline{\mathrm{X}}_{1}(\mathrm{t}) & =\hat{\mathrm{W}}_{1}(\mathrm{t}) \\
\bar{\lambda}_{1}(\mathrm{t}) & =\hat{\mathrm{W}}_{2}(\mathrm{t}) \\
{\left[\begin{array}{l}
\overline{\mathrm{X}}_{2}(\mathrm{t}) \\
\bar{\lambda}_{2}(\mathrm{t})
\end{array}\right] } & =\left[\begin{array}{cc}
\hat{\mathrm{L}}_{11}(\mathrm{t}) & \hat{\mathrm{L}}_{12}(\mathrm{t}) \\
\hat{\mathrm{L}}_{21}(\mathrm{t}) & \hat{\mathrm{L}}_{22}(\mathrm{t})
\end{array}\right]\left[\begin{array}{l}
\overline{\mathrm{X}}_{1}(\mathrm{t}) \\
\bar{\lambda}_{1}(\mathrm{t})
\end{array}\right] .
\end{aligned}
$$




\section{REFERENCES}

[1] Athans, M. and Falb, P.L., "Optimal Control: An Introduction to Theory and Its Application”, McGraw Hill, New York, 1966.

[2] Chang, K.W., "Singular perturbations of a general boundary value problem", SIAM J. Math. Anal. $\underline{3}, 1972$, pp 520-526.

[3] Chang, K.W., "Remarks on a certain hypothesis in singular perturbations", Proc. Amer. Math. Soc., $\underline{23}$, 1969, pp 41-45.

[4] Chang, K.W. and Coppel, W.A, " Singular perturbations of initial value problems over a finite interval”, Arch. Rational Mech. Anal., 32, 1969, pp 268-280.

[5] Harris, W.A., Jr., "Singularly perturbed boundary value problems revisited", Lecture notes in Mathematics 312, Springer-Verlag, Berlin, Heidelberg, New York, 1972, pp 54-64.

[6] Kathirkamanayagan, M. and Ladde, G.S, “ Diagonalization and Stability of Large-scale Singularly Perturbed Linear Systems”, Journal of Math. Anal. and Appl., Vol. 135 (1988), pp. 38-60.

[7] Kathirkamanayagan, M. and Ladde, G.S," Singularly perturbed boundary value problems (to appear)

[8] Khalil, H.K. and Kokotivic, P.V., "D-stability and multiparameter singular perturbations", SIAM J. Control Optim. 17, 1979, pp 56-65.

[9] Kokotovic, P.V., Khalil, H.K. and O'Reilly, J., "Singular Perturbation Methods in Control: Analysis and Design", Academic Press, New York, 1986.

[10] Kokotovic, P.V. and Yackel, R.A., "Singular perturbation of linear regulators: Basic theorems", IEEE Trans. Automat. Contr., 1972, pp 29-37.

[11] Ladde, G.S. and Siljak, D.D., "Multiparameter singular perturbations of linear systems with multiple time scales”, Automatica, $\underline{19}$, 1983, pp 385-394.

[12] O'Malley, R.E., Jr., "Introduction to singular perturbations", Academic Press, New York, 1974.

[13] O'Malley, R.E., Jr., “Boundary value problems for linear systems of ordinary differential equations involving many small parameters”, J.Math.Mech.,18,1969, pp 835-855.

[14] O’Malley, R.E., Jr., “A Singular Singularly - Perturbed Linear Boundary Value Problaem”, SIAM J. Math. Anal., 10, 1979, pp 695-708.

[15] Smith, R., "Singular perturbation theory an introduction with applications", Cambridge University press, New York, 1985.

[16] Smith, R., "Decoupling and order reduction via the riccati transformation", SIAM Review, 29, March 1987 . 
[17] Wilde, R.R. and Koktovic, P.V., "Optimal open - and closed - loop control of singularly perturbed linear systems”, IEEE Trans. Automat. Contr., 18, 1973, pp 616-625.

[18] Wilde, R.R. and Koktovic, P.V., "A dichotomy in linear control theory", IEEE Trans. Automat. Contr., 17, 1972, pp 245-246.

[19] Wonham, W.M., "On a matrix riccati equation of stochastic control”, SIAM J. Control, $\underline{6}$, 1968, pp 681-697. 


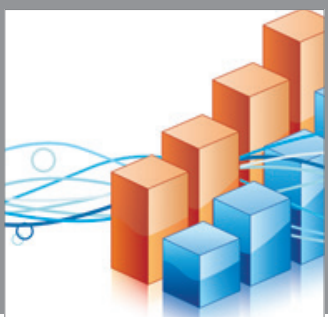

Advances in

Operations Research

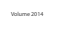

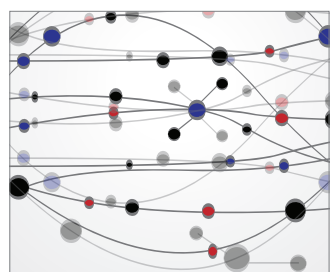

\section{The Scientific} World Journal
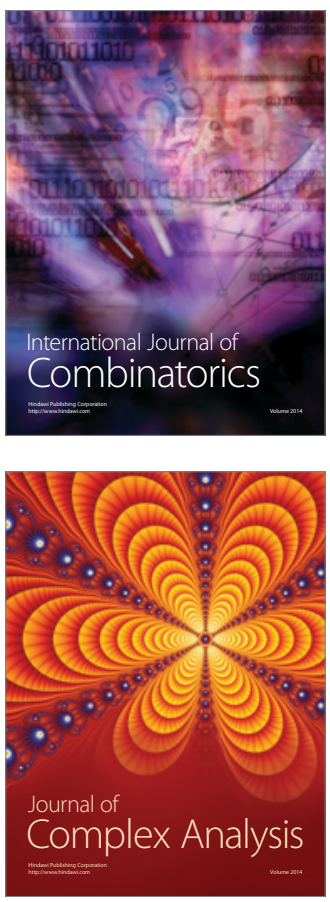

International Journal of

Mathematics and

Mathematical

Sciences
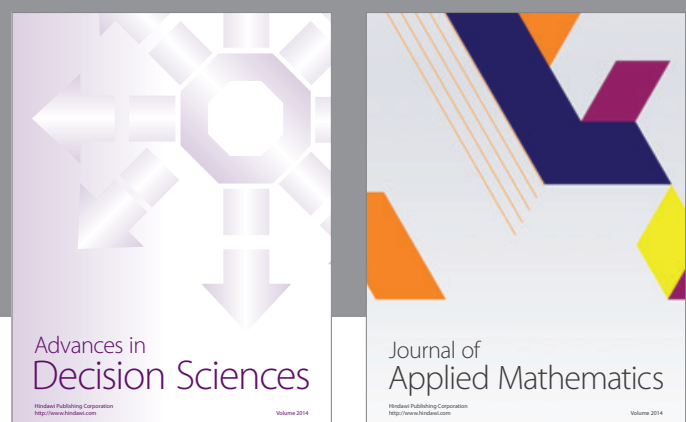

Journal of

Applied Mathematics
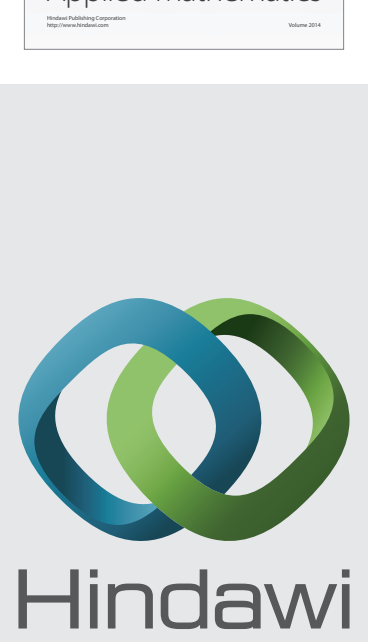

Submit your manuscripts at http://www.hindawi.com
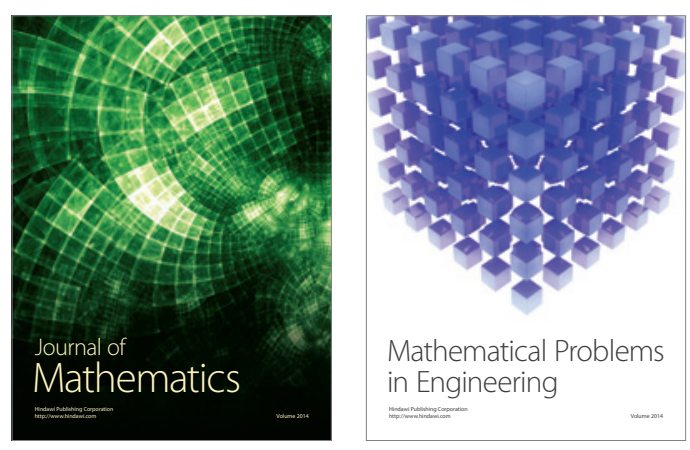

Mathematical Problems in Engineering
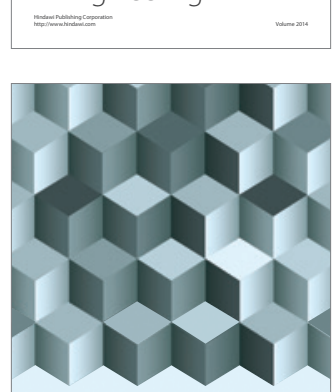

Journal of

Function Spaces
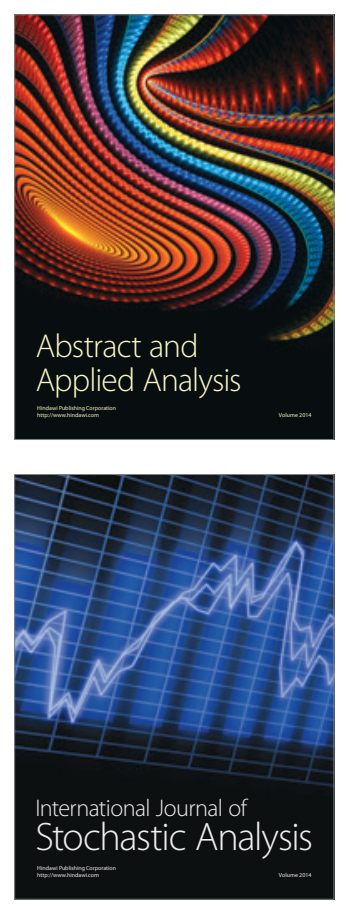

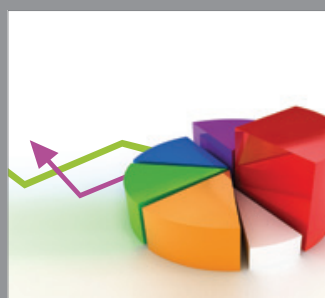

ournal of

Probability and Statistics

Promensencen
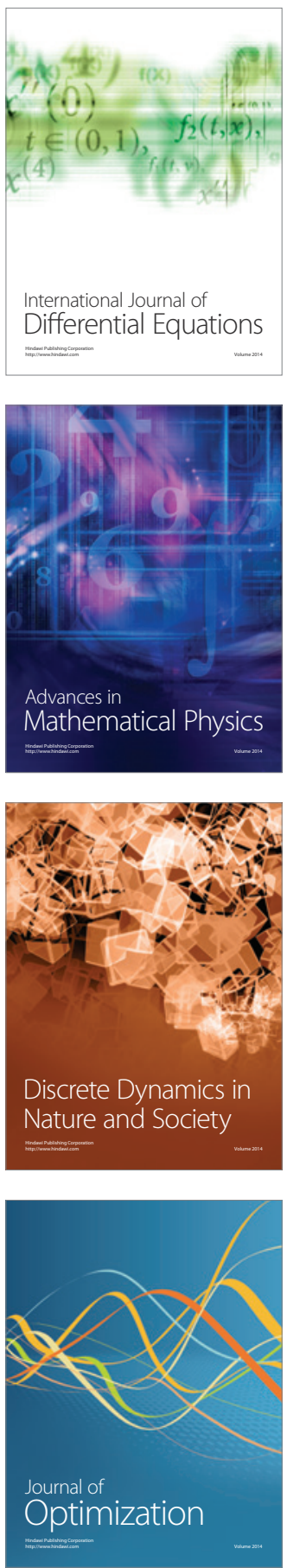